\title{
Assisted Reproductive Technology Surveillance - United States, 2018
}

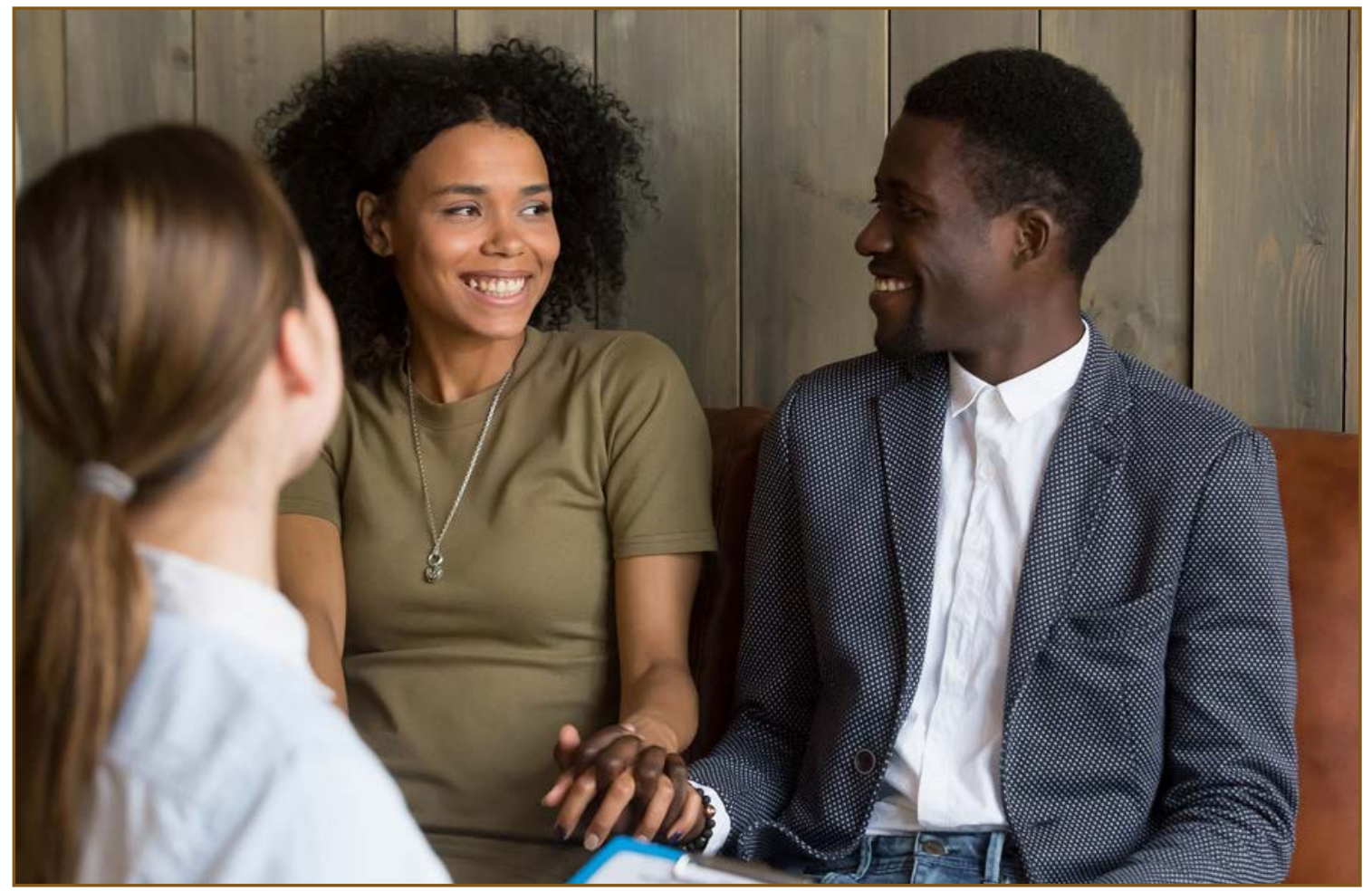




\section{CONTENTS}

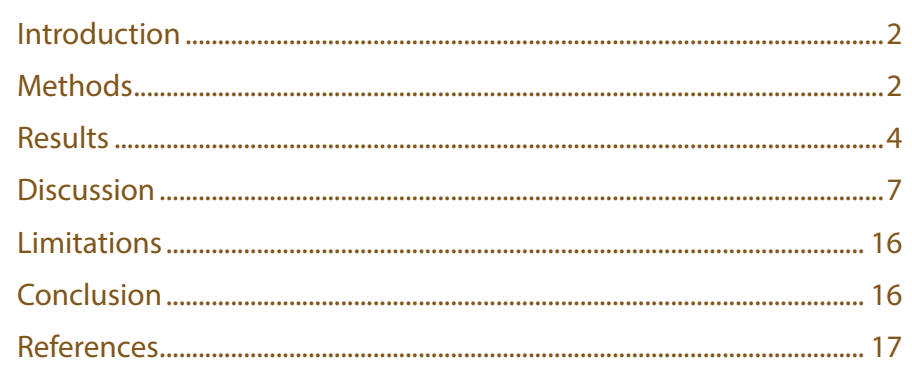

The MMWR series of publications is published by the Center for Surveillance, Epidemiology, and Laboratory Services, Centers for Disease Control and Prevention (CDC), U.S. Department of Health and Human Services, Atlanta, GA 30329-4027.

Suggested citation: [Author names; first three, then et al., if more than six.] [Title]. MMWR Surveill Summ 2022;71(No. SS-\#):[inclusive page numbers].

\section{Centers for Disease Control and Prevention} Rochelle P. Walensky, MD, MPH, Director

Debra Houry, MD, MPH, Acting Principal Deputy Director

Daniel B. Jernigan, MD, MPH, Deputy Director for Public Health Science and Surveillance

Rebecca Bunnell, PhD, MEd, Director, Office of Science

Jennifer Layden, MD, PhD, Deputy Director, Office of Science

Leslie Dauphin, PhD, Acting Director, Center for Surveillance, Epidemiology, and Laboratory Services

\section{MMWR Editorial and Production Staff (Serials)}

Charlotte K. Kent, PhD, MPH, Editor in Chief Christine G. Casey, MD, Editor

Mary Dott, MD, MPH, Online Editor

Terisa F. Rutledge, Managing Editor

David C. Johnson, Lead Technical Writer-Editor Marella Meadows, Project Editor

Matthew L. Boulton, MD, MPH

Carolyn Brooks, ScD, MA

Jay C. Butler, MD

Virginia A. Caine, MD

Jonathan E. Fielding, MD, MPH, MBA

David W. Fleming, MD
Martha F. Boyd, Lead Visual Information Specialist Alexander J. Gottardy, Maureen A. Leahy,

Julia C. Martinroe, Stephen R. Spriggs, Tong Yang, Visual Information Specialists

Quang M. Doan, MBA, Phyllis H. King, Terraye M. Starr, Moua Yang, Information Technology Specialists

MMWR Editorial Board

Timothy F. Jones, MD, Chairman

William E. Halperin, MD, DrPH, MPH

Jewel Mullen, MD, MPH, MPA

Jeff Niederdeppe, $\mathrm{PhD}$

Celeste Philip, MD, MPH

Patricia Quinlisk, MD, MPH

Patrick L. Remington, MD, MPH
Ian Branam, MA,

Acting Lead Health Communication Specialist Shelton Bartley, MPH, Leslie Hamlin, Lowery Johnson, Amanda Ray, Health Communication Specialist Will Yang, MA,

Visual Information Specialist 


\title{
Assisted Reproductive Technology Surveillance - United States, 2018
}

\author{
Saswati Sunderam, $\mathrm{PhD}^{1}$; Dmitry M. Kissin, $\mathrm{MD}^{1}$; Yujia Zhang, $\mathrm{PhD}^{1}$; Amy Jewett, $\mathrm{MPH}^{1}$; Sheree L. Boulet, DrPH ${ }^{2}$, Lee Warner, PhD ${ }^{1}$; \\ Charlan D. Kroelinger, $\mathrm{PhD}^{1}$; Wanda D. Barfield, $\mathrm{MD}^{1}$ \\ ${ }^{1}$ Division of Reproductive Health, National Center for Chronic Disease Prevention and Health Promotion, CDC; ${ }^{2}$ Emory University, School of Medicine, Atlanta, Georgia
}

\section{Abstract}

Problem/Condition: Since the first U.S. infant conceived with assisted reproductive technology (ART) was born in 1981, both the use of ART and the number of fertility clinics providing ART services have increased steadily in the United States. ART includes fertility treatments in which eggs or embryos are handled in the laboratory (i.e., in vitro fertilization [IVF] and related procedures). Although the majority of infants conceived through ART are singletons, women who undergo ART procedures are more likely than women who conceive naturally to have multiple births because multiple embryos might be transferred. Multiple births can pose substantial risks for both mothers and infants, including obstetric complications, preterm birth $(<37$ weeks), and low birthweight $(<2,500 \mathrm{~g})$. This report provides state-specific information for the United States (including the District of Columbia and Puerto Rico) on ART procedures performed in 2018 and compares birth outcomes that occurred in 2018 (resulting from ART procedures performed in 2017 and 2018) with outcomes for all infants born in the United States in 2018.

Period Covered: 2018.

Description of System: In 1995, CDC began collecting data on ART procedures performed in fertility clinics in the United States as mandated by the Fertility Clinic Success Rate and Certification Act of 1992 (Public Law 102-493 [October 24, 1992]). Data are collected through the National ART Surveillance System (NASS), a web-based data collection system developed by CDC. This report includes data from the 50 U.S. states, the District of Columbia, and Puerto Rico.

Results: In 2018, a total of 203,119 ART procedures (range: 196 in Alaska to 26,028 in California) were performed in 456 U.S. fertility clinics and reported to CDC. These procedures resulted in 73,831 live-birth deliveries (range: 76 in Puerto Rico and Wyoming to 9,666 in California) and 81,478 infants born (range: 84 in Wyoming to 10,620 in California). Nationally, among women aged 15-44 years, the rate of ART procedures performed was 3,135 per 1 million women. ART use exceeded 1.5 times the national rate in seven states (Connecticut, Illinois, Maryland, Massachusetts, New Jersey, New York, and Rhode Island) and the District of Columbia. ART use rates exceeded the national rate in an additional seven states (California, Delaware, Hawaii, New Hampshire, Utah, Vermont, and Virginia).

Nationally, among all ART transfer procedures, the average number of embryos transferred was similar across age groups (1.3 among women aged $<35$ years, 1.3 among women aged 35-37 years, and 1.4 among women aged $>37$ years). The national singleembryo transfer (SET) rate among all embryo-transfer procedures was $74.1 \%$ among women aged $<35$ years (range: $28.2 \%$ in Puerto Rico to $89.5 \%$ in Delaware), $72.8 \%$ among women aged $35-37$ years (range: $30.6 \%$ in Puerto Rico to $93.7 \%$ in Delaware), and $66.4 \%$ among women aged $>37$ years (range: $27.1 \%$ in Puerto Rico to $85.3 \%$ in Delaware).

In 2018, ART contributed to 2.0\% of all infants born in the United States (range: $0.4 \%$ in Puerto Rico to 5.1\% in Massachusetts) from procedures performed in 2017 and 2018. Approximately 78.6\% of ART-conceived infants were singleton infants. Overall, ART contributed to $12.5 \%$ of all multiple births, including $12.5 \%$ of all twin births and $13.3 \%$ of all triplets and higher-order births. ART-conceived twins accounted for approximately $97.1 \%$ (15,532 of 16,001) of all ART-conceived multiple births. The percentage of multiple births was higher among infants conceived with ART (21.4\%) than among all infants born in the total birth population (3.3\%). Approximately $20.7 \%(15,532$ of 74,926$)$ of ART-conceived infants were twins, and $0.6 \%$ (469 of 74,926) were triplets and higher-order multiples.

Nationally, infants conceived with ART contributed to $4.2 \%$ of all low birthweight $(<2,500 \mathrm{~g})$ infants. Among ART-conceived infants, $18.3 \%$ were low birthweight compared with $8.3 \%$ among all infants. ART-conceived infants contributed to $5.1 \%$ of

Corresponding author: Saswati Sunderam, Division of Reproductive Health, National Center for Chronic Disease Prevention and Health Promotion, CDC. Telephone: 770-488-6356; Email: msunderam@cdc.gov. all preterm (gestational age $<37$ weeks) infants. The percentage of preterm births was higher among infants conceived with ART $(26.1 \%)$ than among all infants born in the total birth population $(10.0 \%)$. 
The percentage of low birthweight among singletons was $8.3 \%$ among ART-conceived infants and 6.6\% among all infants born. The percentage of preterm births among ART-conceived singleton infants was $14.9 \%$ compared with $8.3 \%$ among all singleton infants. The percentages of small for gestational age infants was $7.3 \%$ among ART-conceived infants compared with $9.4 \%$ among all infants.

Interpretation: Although singleton infants accounted for the majority of ART-conceived infants, multiple births from ART varied substantially among states and nationally, contributing to $>12 \%$ of all twins, triplets, and higher-order multiple infants born in the United States. Because multiple births are associated with higher rates of prematurity than singleton births, the contribution of ART to poor birth outcomes continues to be noteworthy. Although SET rates increased among all age groups, variations in SET rates among states and territories remained, which might reflect variations in embryo-transfer practices among fertility clinics and might in part account for variations in multiple birth rates among states and territories.

Public Health Action: Reducing the number of embryos transferred and increasing use of SET, when clinically appropriate, can help reduce multiple births and related adverse health consequences for both mothers and infants. Whereas risks to mothers from multiple-birth pregnancy include higher rates of caesarean delivery, gestational hypertension, and gestational diabetes, infants from multiple births are at increased risk for numerous adverse sequelae such as preterm birth, birth defects, and developmental disabilities. Long-term follow-up of ART infants through integration of existing maternal and infant health surveillance systems and registries with data available from NASS might be useful for monitoring adverse outcomes on a population basis.

\section{Introduction}

Since the birth of the first U.S. infant conceived with assisted reproductive technology (ART) in 1981, use of advanced technologies to overcome infertility has increased, as has the number of fertility clinics providing ART services and procedures in the United States (1). In 1992, Congress passed the Fertility Clinic Success Rate and Certification Act (Public Law 102-493 [October 24, 1992]), which requires that all U.S. fertility clinics performing ART procedures report data to CDC annually on every ART procedure performed. CDC initiated data collection in 1995 and in 1997 published the first annual ART Fertility Clinic Success Rates Report (2). The annual ART Fertility Clinic Success Rates Report presents multiple measures of success for ART, including the percentage of ART procedures that result in live-birth deliveries.

Although ART has helped millions of women achieve pregnancy, the treatment is associated with potential health risks for both mothers and infants. Because multiple embryos can be transferred in ART procedures, ART might result in multiple-gestation pregnancies and multiple births (3). Obstetric risks to the mothers from a multiple-birth pregnancy include higher rates of caesarean delivery, maternal hemorrhage, pregnancy-related hypertension, and gestational diabetes (4-7). Risks to the infants include preterm birth, low birthweight, birth defects, developmental disability, and death (8-11). In addition, singleton infants conceived with ART might have a higher risk for low birthweight and prematurity than singletons not conceived with ART (12). However, research published in 2017 suggests that this higher risk might be associated with singleton births resulting from multiple-embryo transfers (13).
This report was compiled from data provided and verified by ART clinics on ART procedures performed in 2018 and reported to CDC's Division of Reproductive Health. Data on the use of ART in 2018 are presented for residents of each U.S. state, the District of Columbia, and Puerto Rico. Statespecific data also are reported for outcomes for infants born in 2018 resulting from ART procedures performed in 2017 and 2018. Specifically, the report presents the contribution of ART to selected outcomes (e.g., multiple births, low birthweight, preterm births, and small for gestational age [SGA]) and compares outcomes among ART-conceived infants with outcomes among all infants born in the United States in 2018.

\section{Methods}

\section{National ART Surveillance System}

In 1995, CDC initiated data collection of ART procedures performed in the United States. ART data are obtained from all fertility clinics in the United States that provide and verify information about the outcomes of the ART cycles through the National ART Surveillance System (NASS), a web-based data collection system developed by CDC (https://www.cdc. gov/art/nass/index.html). Clinics that are members of the Society for Assisted Reproductive Technology (SART) can report their data to NASS through SART. Clinics that are not members of SART can enter their data directly in NASS. All clinics must verify the accuracy of the data they report before finalizing submission to NASS. The data then are compiled by a CDC contractor and reviewed for accuracy. In 2018, a total of $8.6 \%$ of clinics did not report their data to CDC and are listed as nonreporting clinics in the 2018 ART Fertility Clinic 
Success Rates Report, as required by the Fertility Clinic Success Rate and Certification Act of 1992. More details about the law are available at https://www.cdc.gov/art/nass/policy.html. Because nonreporting clinics tend to be smaller on average than reporting clinics, NASS is estimated to contain information on 98\% of all ART procedures in the United States (1).

Data collected include patient demographics, medical history, and infertility diagnoses; clinical information pertaining to the ART procedure type; and information about resultant pregnancies and births. The data file contains one record per ART procedure (i.e., cycle of treatment performed). Because ART providers typically do not provide continued prenatal care after a pregnancy is established, information on live births is collected for all procedures from patients and other physicians. CDC's NASS data are available to external researchers through the Collaborative for ART Epidemiologic Research (https:// www.cdc.gov/art/nass/accessdata.html).

\section{ART Procedures}

ART includes fertility treatments in which eggs or embryos are handled in a laboratory (i.e., in vitro fertilization [IVF], gamete intrafallopian transfer, and zygote intrafallopian transfer). More than 99\% of ART procedures performed are IVF. Because an ART procedure consists of multiple steps over an interval of multiple weeks, a procedure often is referred to as a cycle of treatment. An ART cycle usually begins with drug-induced ovarian stimulation. If eggs are produced, the cycle progresses to the egg-retrieval stage, which involves surgical removal of the eggs from the ovaries. After the eggs are retrieved, they are combined with sperm in a laboratory during the IVF procedure. For most IVF procedures (75.7\% in 2018), a specialized technique (intracytoplasmic sperm injection) is used in which a single sperm is injected directly into the egg (1). If successful fertilization occurs, the most viable embryos (i.e., those that appear morphologically most likely to develop and implant) are selected for transfer back into the uterus. If an embryo implants in the uterus, a clinical pregnancy is diagnosed by the presence of a gestational sac detectable by ultrasound. On average, less than half of the procedures result in a clinical pregnancy. Most pregnancies will progress to a livebirth delivery, defined as the delivery of one or more live-born infants; however, some result in pregnancy loss $(14,15)$. ART does not include treatments in which only sperm are handled (i.e., intrauterine insemination) or procedures in which a woman is administered drugs to stimulate egg production without the intention of having eggs retrieved.

ART procedures are classified based on the source of the egg (patient or donor) and the status of the eggs and embryos. Both fresh and thawed embryos can be derived from fresh or frozen eggs of the patient or donor. Patient and donor embryos can be created using sperm from a partner or donor. ART procedures involving fresh eggs and embryos include an egg-retrieval stage. ART procedures that use thawed eggs or embryos do not include egg retrieval because the eggs were retrieved during a previous ART procedure, and the eggs were either frozen or fertilized and the resultant embryos were frozen until the current ART procedure. An ART cycle can be discontinued at any step for medical reasons or by patient choice.

\section{Birth Data for United States}

Data on the total numbers of live births, including singleton and multiple births, in each area in 2018 were obtained from U.S. natality data collected via the National Center for Health Statistics $(16,17)$. The data were derived from birth certificates.

\section{Variables and Definitions}

Data on ART procedures and birth outcomes are presented by patient's residence (i.e., state or territory) at the time of treatment, which might not be the same as the location where the procedure was performed. If information on a patient's residence was missing, residence was assigned as the location where the procedure was performed $(0.3 \%$ of procedures performed in 2018 and $0.1 \%$ of live-birth deliveries occurring in 2018). ART procedures performed in the United States among non-U.S. residents are included in NASS data; however, they are excluded from certain calculations because these women might have delivered outside the United States, and the appropriate denominators were not available. To protect confidentiality, table cells with values of $1-4$ for ART-conceived infants and 0-9 for all infants are suppressed. The cell suppression criteria for ART population allows for the representation of some clinics that carry out only a small number of cycles while maintaining minimum risks for identification. Because of limited numbers, ART data from U.S. territories (with the exception of Puerto Rico) are not included in this report. In addition, percentages derived from cell values $<20$ in the denominator have been suppressed because they are unstable.

This report presents data on all procedures performed with the exception of research cycles and cycles in which egg or embryo banking was performed for future ART cycles. The number of ART procedures performed per 1 million women aged 15-44 years was calculated. Data about population size were compiled based on July 1,2018, estimates from the U.S. Census Bureau (18). The resulting rate approximates the proportion of women who used ART in each state or territory. This proxy measure of ART use is only an approximation because certain women who use ART are outside the age range of $15-44$ years (approximately $5.4 \%$ of 
cycles performed in 2018), and certain women might have had more than one procedure during the reporting period.

A live-birth delivery was defined as a birth of one or more infants. A singleton live-birth delivery was defined as a delivery of only one infant who was born live. A multiple live-birth delivery was defined as a delivery of two or more infants, at least one of whom was born live. Low birthweight was defined as $<2,500 \mathrm{~g}$, moderately low birthweight as $1,500-2,499 \mathrm{~g}$, and very low birthweight as $<1,500 \mathrm{~g}$. Gestational age for births among women who did not undergo ART procedures was calculated using obstetric estimate of gestational age at delivery (19). For births to women who underwent fresh ART procedures, gestational age was calculated by subtracting the date of egg retrieval from the birth date and adding 14 days. For births to women who underwent frozen embryo cycles or fresh ART procedures for which the date of retrieval was not available, gestational age was calculated by subtracting the date of embryo transfer from the birth date and adding 17 days (to account for an average of 3 days in embryo culture). Preterm birth was defined as gestational age $<37$ weeks, late preterm 34-36 weeks, early preterm $<34$ weeks, and very preterm $<32$ weeks (17).

Single-embryo transfer (SET) procedures among all embryotransfer procedures are reported. In a SET procedure, only one embryo is placed in the uterus per transfer regardless of how many embryos are available. The rate of SET was calculated by dividing the total number of SET procedures by the total number of embryo-transfer procedures performed and reported by the following age groups: $<35$ years, 35-37 years, and $>37$ years. The average number of embryos transferred by age group was calculated by dividing the total number of embryos transferred by the total number of embryo-transfer procedures performed among that age group.

The proportion of ART infants among all births in a particular state or territory was used as a second measure of ART use. The proportion of adverse outcomes among ART-conceived infants (e.g., preterm birth) was calculated by dividing the total number of adverse outcomes among ARTconceived infants by the total number of adverse outcomes among all infants born.

The percentage of infants (ART conceived and all infants) born in a state or territory for each plurality group (singleton, multiple, twin, and triplet and higher-order multiple) was calculated by dividing the number of infants (ART conceived and all infants) in each plurality group by the total number of infants born (ART conceived and all infants). The percentage of infants with low birthweight and preterm birth was calculated only for singleton births for ART-conceived infants and for all infants by dividing the number of low birthweight or preterm infants among singletons by the total number of singleton infants.
In addition, the percentage of singleton infants who were SGA (defined as $<10$ th percentile of birthweight for gestational age week and limited to 22-44 weeks) was calculated using a reference distribution (20). The percentage of singleton SGA infants was calculated for all births by dividing the number of singleton SGA infants in the gestational age category (week) by the total number of singleton infants in that gestational age category for ART-conceived and all infants, respectively.

To assess the proportion of ART births among U.S. births in 2018, ART births were aggregated from two reporting years: 1) infants conceived with ART procedures performed in 2017 and born in 2018 (71.2\% of the live-birth deliveries reported to NASS for 2018) and 2) infants conceived with ART procedures performed in 2018 and born in 2018 (28.8\% of the live-birth deliveries reported to NASS for 2018).

\section{Results}

\section{Overview of Fertility Clinics}

In 2018, a total of 499 fertility clinics in the United States performed ART procedures and $456(91.4 \%)$ provided data to CDC, with the majority located in or near major cities (1). The number of fertility clinics performing ART procedures varied by state or territory. The states with the largest numbers of fertility clinics providing data were California (71), Texas (42), and New York (42) (Figure 1).

\section{Number and Type of ART Procedures}

The number, type, and outcome of ART procedures performed in 2018 are provided according to patient's residence for all 52 areas and non-U.S. residents (Table 1). Residence data were missing for approximately $0.3 \%$ of procedures performed, and in these instances, the patient's residence was assigned as the location where the ART procedure was performed. In 2018, approximately $14.2 \%$ of ART procedures were performed in a state or territory other than the patient's state or territory of residence. Non-U.S. residents accounted for approximately $3.6 \%$ of ART procedures, $4.3 \%$ of ART live-birth deliveries, and $4.4 \%$ of ART-conceived infants born.

In 2018, a total of 306,197 ART procedures were reported to CDC (1). This report includes data for 203,119 ART procedures performed (range: 196 in Alaska to 26,028 in California) in the United States (including Puerto Rico) (Table 1) (Figure 2). Excluded are 103,078 cycles in which egg or embryo banking was performed and eight research cycles in which a new treatment procedure was being evaluated. Of 203,119 procedures performed in the 52 states or territories, $163,889(80.7 \%)$ progressed to embryo transfer. Of 163,889 ART procedures that progressed to the embryo-transfer 
FIGURE 1. Location and number* of assisted reproductive technology clinics, by quartile - United States and Puerto Rico, 2018

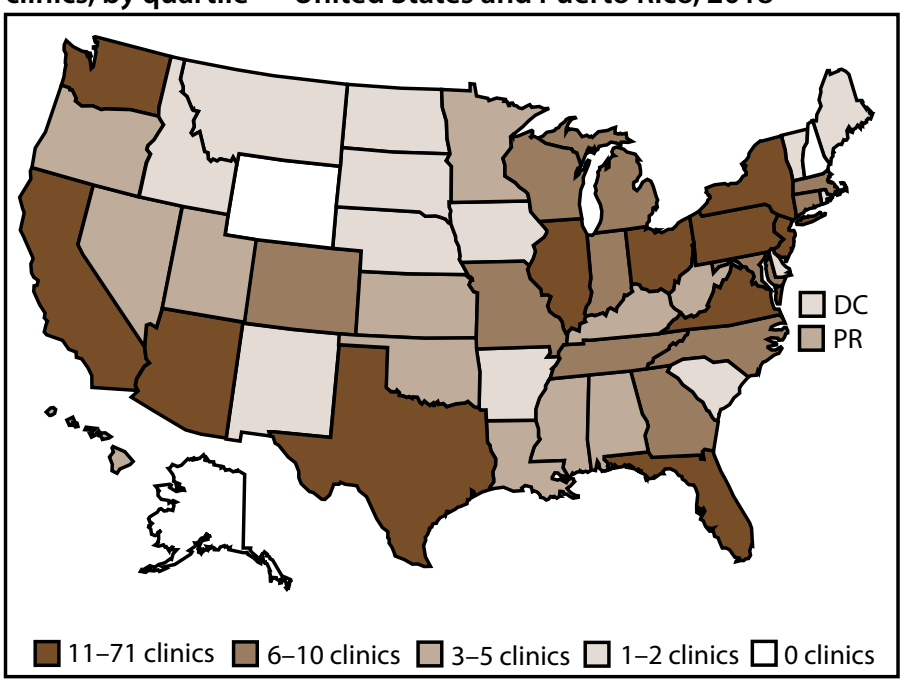

Abbreviations: $\mathrm{DC}=$ District of Columbia; $\mathrm{PR}=$ Puerto Rico.

* In 2018, of the 499 clinics in the United States, 456 (91.4\%) submitted data.

stage, 89,688 (54.7\%) resulted in a pregnancy and 73,831 (45.0\%) in a live-birth delivery (range: 76 in Puerto Rico and Wyoming to 9,666 in California). The 73,831 live-birth deliveries included 66,290 singleton deliveries $(89.8 \%)$ and 7,541 multiple deliveries $(10.2 \%)$ and resulted in 81,478 live-born infants (range: 84 in Wyoming to 10,620 in California).

Six states with the largest numbers of ART procedures (California, Illinois, Massachusetts, New Jersey, New York, and Texas) accounted for approximately half $(47.8 \% ; 97,183$ of 203,119$)$ of all ART procedures, $47.6 \%(78,032$ of 163,889$)$ of all embryo-transfer procedures, $46.1 \%(37,568$ of 81,478$)$ of all ART-conceived infants born, and $41.2 \%(3,106$ of 7,541$)$ of all ART-conceived multiple live-birth deliveries in the United States (Table 1). However, these six states accounted for only $36.1 \%$ of all U.S. births (17).

The number of ART procedures per 1 million women aged 15-44 years ranged from 484 in Puerto Rico to 7,438 in Massachusetts, with an overall national rate of 3,135 (Table 1). Fourteen states (California, Connecticut, Delaware, Hawaii, Illinois, Maryland, Massachusetts, New Hampshire, New Jersey, New York, Rhode Island, Utah, Vermont, and Virginia) and the District of Columbia had ART use rates higher than the national rate. The District of Columbia $(7,082)$ and Massachusetts $(7,438)$ had rates exceeding twice the national rate, whereas Connecticut $(5,312)$, Illinois $(5,120)$, Maryland $(5,518)$, New Jersey $(5,901)$, New York $(5,832)$, and Rhode Island $(4,935)$ had rates exceeding 1.5 times the national rate. The three areas with the lowest ART use rates were Arkansas $(1,014)$, New Mexico (991), and Puerto Rico (484).

\section{Number of Embryos Transferred}

Overall, 66,732 embryo-transfer procedures were performed among women aged $<35$ years, 37,604 among women aged 35-37 years, and 59,553 among women aged $>37$ years (Table 2). Nationally, on average, 1.3 embryos were transferred per procedure among women aged $<35$ years, 1.3 embryos among women aged 35-37 years, and 1.4 embryos among women aged $>37$ years. The national SET rate was $74.1 \%$ among women aged $<35$ years (range: $28.2 \%$ in Puerto Rico to $89.5 \%$ in Delaware), $72.8 \%$ among women aged 35-37 years (range: $30.6 \%$ in Puerto Rico to $93.7 \%$ in Delaware), and $66.4 \%$ among women aged $>37$ years (range: $27.1 \%$ in Puerto Rico to $85.3 \%$ in Delaware).

\section{Singleton and Multiple Births}

To allow comparisons between ART infants and all infants born in the United States, this report includes all ART infants born in 2018. These infants resulted from ART procedures performed in 2017 or 2018.

In 2018, among 3,813,136 infants born in the United States and Puerto Rico, 74,926 (2.0\%) were conceived with ART procedures performed in 2017 and 2018 (Table 3). California, New York, and Texas had the highest total numbers of all infants born (454,920; 226,238; and 378,624, respectively) and ART-conceived infants born (10,016; 7,742; and 6,173, respectively). The percentage of ART-conceived infants among all infants born was highest in Massachusetts (5.1\%), followed by the District of Columbia (4.6\%) and New Jersey (4.2\%).

Nationally, $21.4 \%$ of ART-conceived infants were born in multiple-birth deliveries (range: $6.1 \%$ in Delaware to $45.2 \%$ in Puerto Rico), compared with $3.3 \%$ of all infants (range: $2.1 \%$ in Puerto Rico to $3.7 \%$ in Connecticut, Michigan, and Nebraska) (Table 4). ART-conceived twins accounted for approximately $97.1 \%(15,532$ of 16,001$)$ of all ART-conceived infants born in multiple-birth deliveries. ART-conceived multiple births contributed to $12.5 \%$ of all multiple births (range: $3.7 \%$ in Mississippi to $28.5 \%$ in Hawaii). Approximately $20.7 \%$ of all ART-conceived infants were twins, compared with 3.3\% of all infants. ART-conceived twins contributed to $12.5 \%$ of all twins. Of ART-conceived infants, $0.6 \%$ were triplets and higher-order multiples, compared with $0.1 \%$ among all infants. ART-conceived triplets and higher-order multiples contributed to $13.3 \%$ of all triplets and higher-order multiples.

\section{Adverse Perinatal Outcomes}

Nationally, ART-conceived infants contributed to $4.2 \%$ of all infants with low birthweight, $4.2 \%$ of all infants with moderately low birthweight, and $4.1 \%$ of all infants with very low birthweight 
TABLE 1. Number and outcomes of assisted reproductive technology procedures performed, by female patient's area of residence at time of treatment — United States and Puerto Rico, 2018

\begin{tabular}{|c|c|c|c|c|c|c|c|c|c|}
\hline $\begin{array}{l}\text { Patient's area of } \\
\text { residence* }\end{array}$ & $\begin{array}{l}\text { No. of } \\
\text { ART } \\
\text { clinics }^{\dagger}\end{array}$ & $\begin{array}{l}\text { No. of ART } \\
\text { procedures } \\
\text { performed }^{\S}\end{array}$ & $\begin{array}{l}\text { No. of ART } \\
\text { embryo-transfer } \\
\text { procedures }\end{array}$ & $\begin{array}{l}\text { No. of ART } \\
\text { pregnancies }\end{array}$ & $\begin{array}{l}\text { No. of ART } \\
\text { live-birth } \\
\text { deliveries }\end{array}$ & $\begin{array}{c}\text { No. of ART } \\
\text { singleton live- } \\
\text { birth deliveries }\end{array}$ & $\begin{array}{c}\text { No. of ART } \\
\text { multiple live- } \\
\text { birth deliveries }\end{array}$ & $\begin{array}{l}\text { No. of ART } \\
\text { live-born } \\
\text { infants }\end{array}$ & $\begin{array}{l}\text { No. of ART procedures } \\
\text { per } 1 \text { million women } \\
\text { aged } 15-44 \text { yrs }^{* *}\end{array}$ \\
\hline Alabama & 5 & 1,298 & 973 & 471 & 388 & 353 & 35 & 423 & 1,368 \\
\hline Alaska & 0 & 196 & 159 & 95 & 80 & 71 & 9 & 89 & 1,346 \\
\hline Arizona & 15 & 3,160 & 2,602 & 1,436 & 1,189 & 1,001 & 188 & 1,383 & 2,295 \\
\hline Arkansas & 1 & 586 & 490 & 253 & 210 & 173 & 37 & 249 & 1,014 \\
\hline California & 71 & 26,028 & 20,100 & 11,562 & 9,666 & 8,721 & 945 & 10,620 & 3,218 \\
\hline Colorado & 8 & 2,397 & 2,216 & 1,479 & 1,261 & 1,119 & 142 & 1,404 & 2,063 \\
\hline Connecticut & 6 & 3,558 & 2,812 & 1,623 & 1,343 & 1,210 & 133 & 1,480 & 5,312 \\
\hline Delaware & 2 & 703 & 524 & 319 & 270 & 259 & 11 & 281 & 3,911 \\
\hline District of Columbia & 2 & 1,338 & 1,017 & 540 & 438 & 412 & 26 & 465 & 7,082 \\
\hline Florida & 27 & 9,011 & 7,146 & 3,771 & 3,103 & 2,765 & 338 & 3,447 & 2,325 \\
\hline Georgia & 9 & 4,527 & 3,814 & 2,097 & 1,706 & 1,527 & 179 & 1,889 & 2,093 \\
\hline Hawaii & 6 & 1,078 & 834 & 495 & 403 & 329 & 74 & 480 & 4,075 \\
\hline Idaho & 1 & 611 & 509 & 306 & 272 & 232 & 40 & 312 & 1,810 \\
\hline Illinois ${ }^{\dagger \dagger}$ & 26 & 12,884 & 9,896 & 5,208 & 4,217 & 3,819 & 398 & 4,620 & 5,120 \\
\hline Indiana & 8 & 2,453 & 1,967 & 927 & 779 & 662 & 117 & 899 & 1,888 \\
\hline lowa & 2 & 1,560 & 1,303 & 778 & 647 & 581 & 66 & 715 & 2,622 \\
\hline Kansas & 3 & 970 & 802 & 461 & 383 & 347 & 36 & 420 & 1,732 \\
\hline Kentucky & 4 & 1,387 & 1,150 & 546 & 453 & 372 & 81 & 536 & 1,632 \\
\hline Louisiana & 5 & 1,541 & 1,224 & 632 & 502 & 462 & 40 & 542 & 1,663 \\
\hline Maine & 1 & 493 & 422 & 226 & 192 & 170 & 22 & 215 & 2,133 \\
\hline Maryland & 7 & 6,551 & 5,061 & 2,666 & 2,071 & 1,926 & 145 & 2,218 & 5,518 \\
\hline Massachusetts ${ }^{\dagger \dagger}$ & 8 & 10,390 & 8,633 & 4,250 & 3,469 & 3,230 & 239 & 3,711 & 7,438 \\
\hline Michigan & 10 & 4,400 & 3,682 & 2,018 & 1,641 & 1,382 & 259 & 1,909 & 2,338 \\
\hline Minnesota & 5 & 3,088 & 2,656 & 1,505 & 1,289 & 1,140 & 149 & 1,440 & 2,875 \\
\hline Mississippi & 3 & 628 & 542 & 294 & 243 & 222 & 21 & 265 & 1,065 \\
\hline Missouri & 10 & 2,447 & 2,018 & 1,134 & 933 & 796 & 137 & 1,071 & 2,084 \\
\hline Montana & 1 & 289 & 232 & 143 & 114 & 106 & 8 & 123 & 1,495 \\
\hline Nebraska & 2 & 919 & 724 & 407 & 339 & 277 & 62 & 401 & 2,467 \\
\hline Nevada & 5 & 1,307 & 1,038 & 630 & 527 & 442 & 85 & 614 & 2,199 \\
\hline New Hampshire & 0 & 937 & 791 & 391 & 314 & 279 & 35 & 350 & 3,832 \\
\hline New Jersey ${ }^{\dagger \dagger}$ & 19 & 9,942 & 8,547 & 4,797 & 3,964 & 3,727 & 237 & 4,206 & 5,901 \\
\hline New Mexico & 2 & 394 & 350 & 207 & 169 & 150 & 19 & 188 & 991 \\
\hline New York & 42 & 22,779 & 18,327 & 9,020 & 7,220 & 6,603 & 617 & 7,839 & 5,832 \\
\hline North Carolina & 10 & 4,331 & 3,446 & 2,020 & 1,665 & 1,504 & 161 & 1,830 & 2,126 \\
\hline North Dakota & 1 & 245 & 221 & 140 & 118 & 92 & 26 & 143 & 1,662 \\
\hline Ohio & 11 & 4,988 & 3,976 & 2,117 & 1,749 & 1,585 & 164 & 1,917 & 2,256 \\
\hline Oklahoma & 3 & 1,025 & 840 & 446 & 376 & 310 & 66 & 440 & 1,330 \\
\hline Oregon & 4 & 1,332 & 1,213 & 822 & 699 & 596 & 103 & 804 & 1,623 \\
\hline Pennsylvania & 14 & 7,406 & 5,888 & 2,948 & 2,414 & 2,245 & 169 & 2,585 & 3,107 \\
\hline Puerto Rico & 3 & 298 & 258 & 106 & 76 & 55 & 21 & 98 & 484 \\
\hline Rhode Island ${ }^{\dagger+}$ & 1 & 1,026 & 865 & 351 & 276 & 245 & 31 & 308 & 4,935 \\
\hline South Carolina & 4 & 1,946 & 1,510 & 906 & 729 & 652 & 77 & 809 & 1,997 \\
\hline South Dakota & 1 & 304 & 264 & 136 & 119 & 91 & 28 & 147 & 1,882 \\
\hline Tennessee & 10 & 1,987 & 1,634 & 913 & 767 & 692 & 75 & 843 & 1,503 \\
\hline Texas & 42 & 15,160 & 12,529 & 7,134 & 5,895 & 5,225 & 670 & 6,572 & 2,537 \\
\hline Utah & 3 & 2,180 & 1,833 & 1,106 & 914 & 800 & 114 & 1,026 & 3,158 \\
\hline Vermont & 2 & 435 & 345 & 180 & 148 & 133 & 15 & 163 & 3,780 \\
\hline Virginia & 11 & 6,252 & 4,958 & 2,676 & 2,194 & 1,997 & 197 & 2,390 & 3,703 \\
\hline Washington & 11 & 4,171 & 3,363 & 2,053 & 1,707 & 1,566 & 141 & 1,849 & 2,785 \\
\hline West Virginia & 3 & 409 & 340 & 176 & 142 & 121 & 21 & 165 & 1,282 \\
\hline Wisconsin & 6 & 2,199 & 1,818 & 956 & 818 & 719 & 99 & 918 & 2,022 \\
\hline Wyoming & 0 & 209 & 176 & 104 & 76 & 68 & 8 & 84 & 1,944 \\
\hline Non-U.S. residents & - & 7,366 & 5,851 & 3,711 & 3,154 & 2,729 & 425 & 3,583 & _§§ \\
\hline Total & 456 & 203,119 & 163,889 & 89,688 & 73,831 & 66,290 & 7,541 & 81,478 & 3,135 \\
\hline
\end{tabular}

Abbreviation: ART = assisted reproductive technology.

* In cases of missing patient's residence data $(0.3 \%)$, the patient's residence was assigned as the location where the ART procedure was performed.

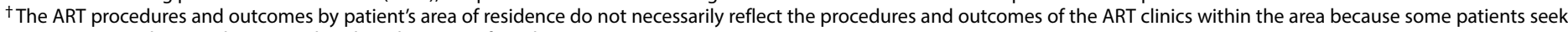
treatment at a clinic in a location other than their area of residence.

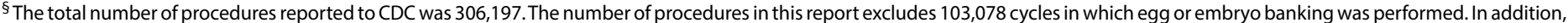
all numbers and percentages exclude eight research cycles that were evaluating new procedures.

"Embryo-transfer procedures include all procedures performed in which at least one embryo was transferred.

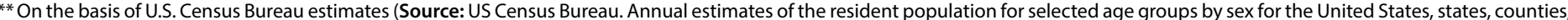

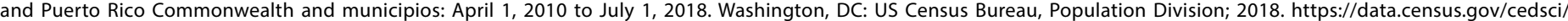
table?q=United\%20States\&g=0100000US\&tid=ACSST1Y2018.S0101\&vintage $=2018$ ).

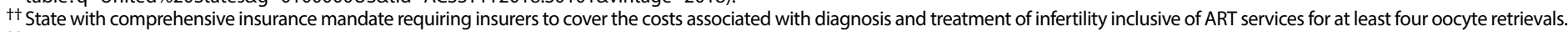

$\S \S$ Non-U.S. residents were excluded from rate because the appropriate denominators were not available. 
FIGURE 2. Number and outcomes of assisted reproductive technology procedures performed, by type of outcome - United States and Puerto Rico, 2018

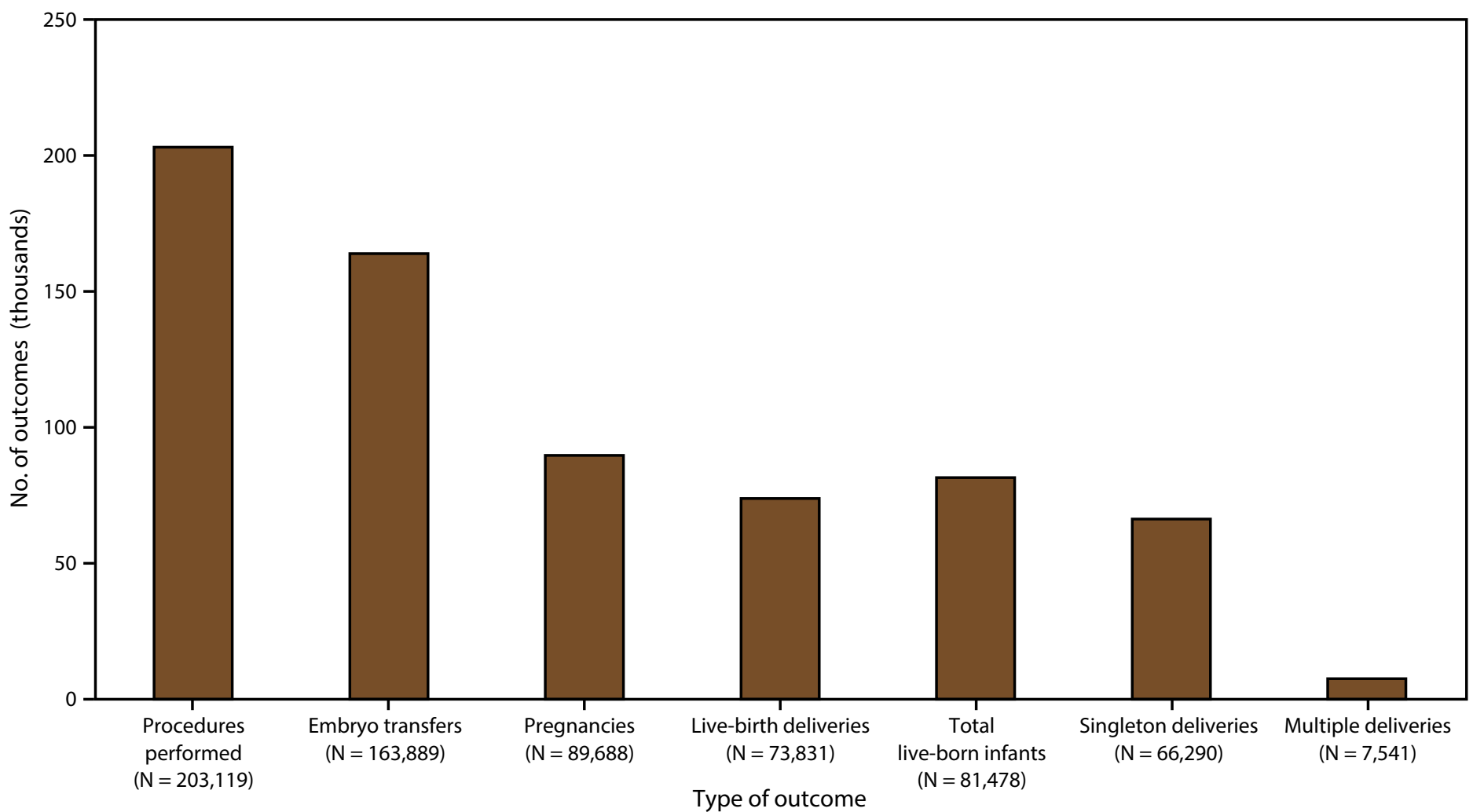

* The total number of procedures reported to CDC was 306,197. Procedures performed exclude 103,078 cycles in which egg or embryo banking was performed and eight research cycles in which a new treatment procedure was being evaluated. Embryo transfers include all procedures in which at least one embryo was transferred.

(Table 5). Among all ART-conceived infants (including multiples), $18.3 \%$ had low birthweight compared with $8.3 \%$ among all infants (including multiples). Approximately $3.0 \%$ of all ARTconceived infants (including multiples) had very low birthweight compared with $1.4 \%$ among all infants (including multiples).

Nationally, ART contributed to approximately $5.1 \%$ of all infants born preterm, $5.0 \%$ very preterm, $5.5 \%$ early preterm, and $4.9 \%$ late preterm (Table 6). In Connecticut and Massachusetts, the contribution of ART to preterm infants was $\geq 10 \%$. Among all ART-conceived infants (including multiples), 26.1\% had born preterm, $4.0 \%$ very preterm, $7.8 \%$ early preterm, and $18.4 \%$ late preterm. Corresponding rates among all infants (including multiples) were $10.0 \%$ preterm, $1.6 \%$ very preterm, $2.8 \%$ early preterm, and $7.3 \%$ late preterm. Late preterm births accounted for the majority of preterm births among both ART-conceived infants and all infants $(70.2 \%$ and $72.6 \%$, respectively).

Among singletons only, the percentage of infants who had low birthweight was $8.3 \%$ among ART-conceived infants and $6.6 \%$ among all infants. In addition, among singletons, the percentage of infants who were born preterm was $14.9 \%$ among ART-conceived infants and 8.3\% among all infants, and the percentage of SGA infants was $7.3 \%$ among ARTconceived infants and $9.4 \%$ for all infants (Table 7 ).

\section{Discussion}

\section{Overview}

The use of ART has increased substantially in the United States since the beginning of ART surveillance. In 1996 (the first full year for which ART data were reported to CDC), 20,597 infants were born from 64,036 ART procedures performed by 302 reporting clinics (2). Since then, the number of clinics performing ART services has increased considerably, the number of reported procedures has tripled, and the number of infants born from ART procedures has almost quadrupled. Multiple improvements can be observed in embryo-transfer practices and ART outcomes by comparing data for years 2017 and 2018 (21). The rate of SET procedures increased from $67.3 \%$ to $74.1 \%$ among women aged $<35$ years (a $10.1 \%$ increase), from $65.0 \%$ to $72.8 \%$ among women aged $35-37$ years (a $12.0 \%$ increase), and from $60.0 \%$ to $66.4 \%$ among women aged $>37$ years (a $10.7 \%$ increase). Relatedly, from 2017 to 2018 the percentage of singleton births increased from $73.6 \%$ to $78.6 \%$ (a $6.8 \%$ increase), the percentage of twin births decreased from $25.5 \%$ to $20.7 \%$ (a $18.8 \%$ decrease), and the percentage of triplets and higher-order multiples decreased from $0.9 \%$ to $0.6 \%$ (a $33.3 \%$ decrease). The percentage of 
TABLE 2. Number of assisted reproductive technology embryo-transfer procedures performed with a single-embryo transfer, by female patient's age group and area of residence at time of treatment — United States and Puerto Rico, 2018

\begin{tabular}{|c|c|c|c|c|c|c|c|c|c|}
\hline \multirow[b]{2}{*}{$\begin{array}{l}\text { Patient's area of } \\
\text { residence* }\end{array}$} & \multicolumn{3}{|c|}{$<35$ yrs } & \multicolumn{3}{|c|}{$35-37$ yrs } & \multicolumn{3}{|c|}{$>37$ yrs } \\
\hline & $\begin{array}{c}\text { No. of } \\
\text { embryo-transfer } \\
\text { procedures }^{\dagger}\end{array}$ & $\begin{array}{l}\text { Average no. of } \\
\text { embryos } \\
\text { transferred }\end{array}$ & $\begin{array}{l}\text { SET } \\
(\%)\end{array}$ & $\begin{array}{c}\text { No. of } \\
\text { embryo-transfer } \\
\text { procedures }^{\dagger}\end{array}$ & $\begin{array}{l}\text { Average no. of } \\
\text { embryos transferred }\end{array}$ & $\begin{array}{l}\text { SET } \\
(\%)\end{array}$ & $\begin{array}{l}\text { No. of embryo- } \\
\text { transfer procedures }{ }^{\dagger}\end{array}$ & $\begin{array}{l}\text { Average no. of } \\
\text { embryos transferred }\end{array}$ & $\begin{array}{l}\text { SET } \\
(\%)\end{array}$ \\
\hline Alabama & 513 & 1.3 & 71.0 & 212 & 1.4 & 66.0 & 248 & 1.5 & 57.3 \\
\hline Alaska & 56 & 1.3 & 66.1 & 39 & 1.1 & 87.2 & 64 & 1.4 & 68.8 \\
\hline Arizona & 1,161 & 1.5 & 57.3 & 591 & 1.4 & 61.8 & 850 & 1.5 & 58.1 \\
\hline Arkansas & 262 & 1.4 & 59.9 & 119 & 1.4 & 57.1 & 109 & 1.6 & 52.3 \\
\hline California & 6,016 & 1.3 & 73.9 & 4,920 & 1.3 & 74.5 & 9,164 & 1.4 & 68.6 \\
\hline Colorado & 904 & 1.2 & 76.8 & 546 & 1.2 & 81.0 & 766 & 1.2 & 77.0 \\
\hline Connecticut & 1,109 & 1.2 & 80.7 & 681 & 1.3 & 68.4 & 1,022 & 1.5 & 60.9 \\
\hline Delaware & 266 & 1.1 & 89.5 & 95 & 1.1 & 93.7 & 163 & 1.2 & 85.3 \\
\hline District of Columbia & 226 & 1.1 & 88.1 & 229 & 1.2 & 83.4 & 562 & 1.3 & 76.0 \\
\hline Florida & 2,861 & 1.3 & 70.5 & 1,614 & 1.3 & 70.6 & 2,671 & 1.4 & 67.4 \\
\hline Georgia & 1,598 & 1.3 & 73.2 & 863 & 1.3 & 72.2 & 1,353 & 1.3 & 71.9 \\
\hline Hawaii & 268 & 1.5 & 51.5 & 171 & 1.5 & 55.6 & 395 & 1.7 & 50.4 \\
\hline Idaho & 276 & 1.4 & 60.9 & 76 & 1.3 & 68.4 & 157 & 1.4 & 63.7 \\
\hline Illinois ${ }^{\S}$ & 4,105 & 1.3 & 72.1 & 2,406 & 1.3 & 67.8 & 3,385 & 1.5 & 59.4 \\
\hline Indiana & 1,076 & 1.4 & 62.5 & 439 & 1.4 & 62.2 & 452 & 1.5 & 57.5 \\
\hline lowa & 771 & 1.2 & 75.7 & 261 & 1.3 & 71.3 & 271 & 1.3 & 72.3 \\
\hline Kansas & 420 & 1.2 & 82.6 & 206 & 1.2 & 78.2 & 176 & 1.3 & 78.4 \\
\hline Kentucky & 662 & 1.4 & 63.0 & 213 & 1.4 & 58.7 & 275 & 1.6 & 49.1 \\
\hline Louisiana & 636 & 1.2 & 82.9 & 292 & 1.2 & 79.1 & 296 & 1.3 & 79.1 \\
\hline Maine & 180 & 1.2 & 77.8 & 103 & 1.3 & 75.7 & 139 & 1.3 & 74.1 \\
\hline Maryland & 1,951 & 1.2 & 83.5 & 1,110 & 1.2 & 80.0 & 2,000 & 1.4 & 67.7 \\
\hline Massachusetts ${ }^{\S}$ & 3,319 & 1.1 & 87.5 & 2,138 & 1.2 & 81.5 & 3,176 & 1.6 & 60.1 \\
\hline Michigan & 1,834 & 1.5 & 52.1 & 825 & 1.5 & 54.2 & 1,023 & 1.7 & 52.4 \\
\hline Minnesota & 1,311 & 1.3 & 68.0 & 613 & 1.3 & 71.9 & 732 & 1.4 & 65.3 \\
\hline Mississippi & 316 & 1.3 & 74.4 & 107 & 1.4 & 65.4 & 119 & 1.2 & 75.6 \\
\hline Missouri & 1,104 & 1.4 & 62.0 & 435 & 1.5 & 56.3 & 479 & 1.6 & 58.0 \\
\hline Montana & 115 & 1.2 & 79.1 & 61 & 1.2 & 82.0 & 56 & 1.3 & 76.8 \\
\hline Nebraska & 422 & 1.3 & 66.8 & 149 & 1.4 & 60.4 & 153 & 1.4 & 66.0 \\
\hline Nevada & 471 & 1.4 & 62.2 & 222 & 1.3 & 67.1 & 345 & 1.3 & 73.3 \\
\hline New Hampshire & 363 & 1.2 & 81.0 & 200 & 1.3 & 74.0 & 228 & 1.5 & 64.5 \\
\hline New Jersey ${ }^{\S}$ & 3,303 & 1.2 & 85.4 & 1,951 & 1.2 & 82.6 & 3,293 & 1.3 & 73.9 \\
\hline New Mexico & 150 & 1.3 & 70.0 & 94 & 1.2 & 77.7 & 106 & 1.5 & 62.3 \\
\hline New York & 6,214 & 1.3 & 75.2 & 3,950 & 1.3 & 72.2 & 8,163 & 1.5 & 61.9 \\
\hline North Carolina & 1,593 & 1.2 & 80.7 & 754 & 1.3 & 74.1 & 1,099 & 1.4 & 67.2 \\
\hline North Dakota & 111 & 1.4 & 55.9 & 51 & 1.5 & 52.9 & 59 & 1.4 & 59.3 \\
\hline Ohio & 2,005 & 1.2 & 77.6 & 913 & 1.3 & 70.4 & 1,058 & 1.4 & 61.9 \\
\hline Oklahoma & 442 & 1.5 & 55.0 & 204 & 1.6 & 45.1 & 194 & 1.6 & 47.4 \\
\hline Oregon & 438 & 1.4 & 64.8 & 288 & 1.3 & 69.8 & 487 & 1.3 & 74.7 \\
\hline Pennsylvania & 2,757 & 1.2 & 81.8 & 1,445 & 1.2 & 78.2 & 1,686 & 1.4 & 70.3 \\
\hline Puerto Rico & 78 & 1.8 & 28.2 & 62 & 1.8 & 30.6 & 118 & 1.9 & 27.1 \\
\hline Rhode Island $\$$ & 373 & 1.2 & 82.8 & 209 & 1.2 & 76.6 & 283 & 1.6 & 57.6 \\
\hline South Carolina & 709 & 1.3 & 75.2 & 345 & 1.3 & 66.7 & 456 & 1.4 & 65.6 \\
\hline South Dakota & 171 & 1.6 & 40.4 & 50 & 1.6 & 42.0 & 43 & 1.3 & 69.8 \\
\hline Tennessee & 804 & 1.2 & 78.2 & 347 & 1.3 & 70.9 & 483 & 1.4 & 67.5 \\
\hline Texas & 5,956 & 1.3 & 72.4 & 2,930 & 1.3 & 72.6 & 3,643 & 1.4 & 68.9 \\
\hline Utah & 1,064 & 1.3 & 69.6 & 329 & 1.3 & 67.5 & 440 & 1.4 & 65.2 \\
\hline Vermont & 115 & 1.3 & 73.9 & 75 & 1.4 & 62.7 & 155 & 1.6 & 59.4 \\
\hline Virginia & 1,836 & 1.2 & 79.3 & 1,175 & 1.2 & 78.9 & 1,947 & 1.3 & 72.5 \\
\hline Washington & 1,265 & 1.2 & 79.4 & 840 & 1.2 & 81.3 & 1,258 & 1.3 & 76.9 \\
\hline West Virginia & 193 & 1.5 & 58.0 & 74 & 1.3 & 70.3 & 73 & 1.5 & 63.0 \\
\hline Wisconsin & 918 & 1.3 & 69.9 & 467 & 1.4 & 65.7 & 433 & 1.4 & 63.5 \\
\hline Wyoming & 93 & 1.2 & 78.5 & 39 & 1.3 & 74.4 & 44 & 1.4 & 63.6 \\
\hline Non-U.S. resident & 1,572 & 1.3 & 68.0 & 1,076 & 1.3 & 72.1 & 3,203 & 1.3 & 72.7 \\
\hline Total & 66,732 & 1.3 & 74.1 & 37,604 & 1.3 & 72.8 & 59,553 & 1.4 & 66.4 \\
\hline
\end{tabular}

Abbreviation: $\mathrm{SET}=$ single-embryo transfer.

* In cases of missing patient's residence data $(0.3 \%)$, the patient's residence was assigned as the location where the assisted reproductive technology procedure was performed.

$\dagger$ Includes all procedures in which at least one embryo was transferred.

$\S$ State with comprehensive insurance mandate requiring insurers to cover the costs associated with diagnosis and treatment of infertility inclusive of ART services for at least four oocyte retrievals. 
TABLE 3. Number, proportion, and percentage of infants born with use of assisted reproductive technology, by female patient's area of residence at time of treatment — United States and Puerto Rico, 2018

\begin{tabular}{|c|c|c|c|c|c|c|}
\hline & Total infants born ${ }^{\dagger, \S}$ & $\begin{array}{c}\text { ART infants } \\
\text { born? }\end{array}$ & $\begin{array}{l}\text { Proportion of ART } \\
\text { infants among } \\
\text { all infants }\end{array}$ & $\begin{array}{l}\text { Singleton infants } \\
\text { among ART infants }\end{array}$ & $\begin{array}{l}\text { Singleton infants } \\
\text { among all infants }\end{array}$ & $\begin{array}{l}\text { Proportion of ART } \\
\text { singleton infants among } \\
\text { all singleton infants }\end{array}$ \\
\hline Patient's area of residence* & No. & No. & $\%$ & No. (\%) & No. (\%) & $\%$ \\
\hline Alabama & 57,761 & 389 & 0.7 & $284(73.0)$ & $55,655(96.4)$ & 0.5 \\
\hline Alaska & 10,086 & 76 & 0.8 & 56 (73.7) & 9,741 (96.6) & 0.6 \\
\hline Arizona & 80,723 & 1,329 & 1.6 & $898(67.6)$ & $78,082(96.7)$ & 1.2 \\
\hline Arkansas & 37,018 & 246 & 0.7 & $149(60.6)$ & 35,854 (96.9) & 0.4 \\
\hline California & 454,920 & 10,016 & 2.2 & $8,046(80.3)$ & $440,699(96.9)$ & 1.8 \\
\hline Colorado & 62,885 & 1,418 & 2.3 & $1,097(77.4)$ & $60,867(96.8)$ & 1.8 \\
\hline Connecticut & 34,725 & 1,426 & 4.1 & $1,133(79.5)$ & $33,429(96.3)$ & 3.4 \\
\hline Delaware & 10,621 & 229 & 2.2 & $215(93.9)$ & $10,323(97.2)$ & 2.1 \\
\hline District of Columbia & 9,212 & 428 & 4.6 & $380(88.8)$ & $8,887(96.5)$ & 4.3 \\
\hline Florida & 221,542 & 3,236 & 1.5 & $2,450(75.7)$ & $214,480(96.8)$ & 1.1 \\
\hline Georgia & 126,172 & 1,843 & 1.5 & $1,422(77.2)$ & $121,778(96.5)$ & 1.2 \\
\hline Hawaii & 16,972 & 425 & 2.5 & $274(64.5)$ & $16,443(96.9)$ & 1.7 \\
\hline Idaho & 21,403 & 312 & 1.5 & $203(65.1)$ & $20,690(96.7)$ & 1.0 \\
\hline Illinois** & 144,815 & 4,474 & 3.1 & 3,591 (80.3) & 139,561 (96.4) & 2.6 \\
\hline Indiana & 81,646 & 831 & 1.0 & $598(72.0)$ & 78,876 (96.6) & 0.8 \\
\hline lowa & 37,785 & 689 & 1.8 & $543(78.8)$ & 36,433 (96.4) & 1.5 \\
\hline Kansas & 36,261 & 401 & 1.1 & $333(83.0)$ & $35,097(96.8)$ & 0.9 \\
\hline Kentucky & 53,922 & 533 & 1.0 & $369(69.2)$ & $52,091(96.6)$ & 0.7 \\
\hline Louisiana & 59,615 & 539 & 0.9 & $430(79.8)$ & $57,526(96.5)$ & 0.7 \\
\hline Maine & 12,311 & 206 & 1.7 & $152(73.8)$ & $11,884(96.5)$ & 1.3 \\
\hline Maryland & 71,080 & 2,192 & 3.1 & $1,840(83.9)$ & $68,592(96.5)$ & 2.7 \\
\hline Massachusetts** & 69,109 & 3,543 & 5.1 & 2,996 (84.6) & $66,664(96.5)$ & 4.5 \\
\hline Michigan & 110,032 & 1,778 & 1.6 & $1,194(67.2)$ & $105,973(96.3)$ & 1.1 \\
\hline Minnesota & 67,344 & 1,396 & 2.1 & $1,047(75.0)$ & $65,015(96.5)$ & 1.6 \\
\hline Mississippi & 37,000 & 244 & 0.7 & $196(80.3)$ & $35,697(96.5)$ & 0.5 \\
\hline Missouri & 73,269 & 968 & 1.3 & $670(69.2)$ & $70,611(96.4)$ & 0.9 \\
\hline Montana & 11,513 & 133 & 1.2 & $110(82.7)$ & $11,184(97.1)$ & 1.0 \\
\hline Nebraska & 25,488 & 347 & 1.4 & $239(68.9)$ & $24,551(96.3)$ & 1.0 \\
\hline Nevada & 35,682 & 567 & 1.6 & $421(74.3)$ & $34,638(97.1)$ & 1.2 \\
\hline New Hampshire & 11,995 & 438 & 3.7 & $328(74.9)$ & $11,579(96.5)$ & 2.8 \\
\hline New Jersey** & 101,223 & 4,239 & 4.2 & 3,625 (85.5) & $97,667(96.5)$ & 3.7 \\
\hline New Mexico & 23,039 & 186 & 0.8 & $148(79.6)$ & $22,453(97.5)$ & 0.7 \\
\hline New York & 226,238 & 7,742 & 3.4 & 6,266 (80.9) & $218,244(96.5)$ & 2.9 \\
\hline North Carolina & 118,954 & 1,855 & 1.6 & $1,436(77.4)$ & $114,948(96.6)$ & 1.2 \\
\hline North Dakota & 10,636 & 133 & 1.3 & $90(67.7)$ & $10,290(96.7)$ & 0.9 \\
\hline Ohio & 135,134 & 1,840 & 1.4 & 1,483 (80.6) & 130,554 (96.6) & 1.1 \\
\hline Oklahoma & 49,800 & 393 & 0.8 & $271(69.0)$ & 48,129 (96.6) & 0.6 \\
\hline Oregon & 42,188 & 756 & 1.8 & $534(70.6)$ & $40,698(96.5)$ & 1.3 \\
\hline Pennsylvania & 135,673 & 2,538 & 1.9 & $2,142(84.4)$ & $131,199(96.7)$ & 1.6 \\
\hline Puerto Rico & 21,424 & 84 & 0.4 & $46(54.8)$ & 20,977 (97.9) & 0.2 \\
\hline Rhode Island** & 10,506 & 301 & 2.9 & $240(79.7)$ & $10,123(96.4)$ & 2.4 \\
\hline South Carolina & 56,669 & 692 & 1.2 & $535(77.3)$ & $54,785(96.7)$ & 1.0 \\
\hline South Dakota & 11,893 & 129 & 1.1 & $91(70.5)$ & $11,466(96.4)$ & 0.8 \\
\hline Tennessee & 80,751 & 794 & 1.0 & $601(75.7)$ & 78,042 (96.6) & 0.8 \\
\hline Texas & 378,624 & 6,173 & 1.6 & 4,704 (76.2) & $366,664(96.8)$ & 1.3 \\
\hline Utah & 47,209 & 1,091 & 2.3 & $786(72.0)$ & $45,580(96.5)$ & 1.7 \\
\hline Vermont & 5,432 & 120 & 2.2 & $91(75.8)$ & 5,249 (96.6) & 1.7 \\
\hline Virginia & 99,843 & 2,339 & 2.3 & $1,946(83.2)$ & $96,370(96.5)$ & 2.0 \\
\hline Washington & 86,085 & 1,733 & 2.0 & $1,408(81.2)$ & $83,506(97.0)$ & 1.7 \\
\hline West Virginia & 18,248 & 121 & 0.7 & 87 (71.9) & $17,657(96.8)$ & 0.5 \\
\hline Wisconsin & 64,098 & 917 & 1.4 & $665(72.5)$ & 61,795 (96.4) & 1.1 \\
\hline Wyoming & 6,562 & 98 & 1.5 & $66(67.3)$ & $6,332(96.5)$ & 1.0 \\
\hline Total & $3,813,136$ & 74,926 & 2.0 & $58,925(78.6)$ & $3,685,628(96.7)$ & 1.6 \\
\hline
\end{tabular}

Abbreviation: ART = assisted reproductive technology.

* In cases of missing patient's residence data $(0.3 \%)$, the patient's residence was assigned as the location where the ART procedure was performed.

† U.S. births exclude births to non-U.S. residents (Source: Martin JA, Hamilton BE, Osterman MJ, Driscoll AK. Births: final data for 2018. Natl Vital Stat Rep 2018;68:1-47).

$\S$ U.S. births exclude births to non-U.S. residents (Source: National Center for Health Statistics, Vital statistics data available. Natality public use file and CD-ROM. Hyattsville, MD, National Center for Health Statistics).

I Includes infants conceived from ART procedures performed in 2017 and born in 2018 and infants conceived from ART procedures performed in 2018 and born in 2018. Total ART births exclude births to non-U.S. residents.

** State with comprehensive insurance mandate requiring insurers to cover the costs associated with diagnosis and treatment of infertility inclusive of ART services for at least four oocyte retrievals. 
TABLE 4. Number, percentage, and proportion of multiple-birth infants, twins, and triplets and higher-order infants born with use of assisted reproductive technology procedures, by female patient's area of residence at time of treatment — United States and Puerto Rico, 2018

\begin{tabular}{|c|c|c|c|c|c|c|c|c|c|}
\hline \multirow{2}{*}{$\begin{array}{l}\text { Patient's area of } \\
\text { residence* }\end{array}$} & $\begin{array}{l}\text { Multiple-birth } \\
\text { infants among } \\
\text { ART infants, } \\
\end{array}$ & $\begin{array}{c}\text { Multiple } \\
\text { births among } \\
\text { all infants } \\
\end{array}$ & $\begin{array}{c}\text { Proportion of } \\
\text { ART multiple } \\
\text { births among } \\
\text { all multiple } \\
\text { births } \\
\end{array}$ & $\begin{array}{c}\text { Twin infants } \\
\text { among ART } \\
\text { infants } \text { S }^{\dagger} \S \\
\end{array}$ & $\begin{array}{l}\text { Twin infants } \\
\text { among all } \\
\text { infants } \\
\end{array}$ & $\begin{array}{l}\text { Proportion of } \\
\text { ART twin } \\
\text { infants } \\
\text { among all } \\
\text { twin infants } \\
\end{array}$ & $\begin{array}{c}\text { Triplets and } \\
\text { higher-order } \\
\text { infants } \\
\text { among ART } \\
\text { infants }{ }^{\dagger, \S} \\
\end{array}$ & $\begin{array}{l}\text { Triplets and } \\
\text { higher-order } \\
\text { infants } \\
\text { among all } \\
\text { infants } \\
\end{array}$ & $\begin{array}{l}\text { Proportion of ART } \\
\text { triplets and higher- } \\
\text { order infants among } \\
\text { all triplets and } \\
\text { higher-order infants } \\
\end{array}$ \\
\hline & No. (\%) & No. (\%) & $\%$ & No. (\%) & No. (\%) & $\%$ & No. (\%) & No. (\%) & $\%$ \\
\hline Alabama & $105(27.0)$ & $2,106(3.6)$ & 5.0 & $96(24.7)$ & $2,015(3.5)$ & 4.8 & $9(2.3)$ & $91(0.2)$ & 9.9 \\
\hline Alaska & $20(26.3)$ & $345(3.4)$ & 5.8 & $20(26.3)$ & $330(3.3)$ & 6.1 & $0(0.0)$ & $15(0.1)$ & $-^{\dagger+}$ \\
\hline Arizona & $431(32.4)$ & $2,641(3.3)$ & 16.3 & $412(31.0)$ & $2,545(3.2)$ & 16.2 & $19(1.4)$ & $96(0.1)$ & 19.8 \\
\hline Arkansas & 97 (39.4) & 1,164 (3.1) & 8.3 & $88(35.8)$ & $1,124(3.0)$ & 7.8 & $9(3.7)$ & $40(0.1)$ & 22.5 \\
\hline California & $1,970(19.7)$ & $14,221(3.1)$ & 13.9 & $1,928(19.2)$ & $13,851(3.0)$ & 13.9 & $42(0.4)$ & $370(0.1)$ & 11.4 \\
\hline Colorado & $321(22.6)$ & $2,018(3.2)$ & 15.9 & $321(22.6)$ & $1,990(3.2)$ & 16.1 & $0(0.0)$ & $28(0.0)$ & 0.0 \\
\hline Connecticut & $293(20.5)$ & $1,296(3.7)$ & 22.6 & $287(20.1)$ & $1,265(3.6)$ & 22.7 & $6(0.4)$ & $31(0.1)$ & 19.4 \\
\hline Delaware & $14(6.1)$ & $298(2.8)$ & 4.7 & $14(6.1)$ & - ** & - ${ }^{* *}$ & $0(0.0)$ & - ${ }^{* *}$ & - **,+十 \\
\hline District of Columbia & $48(11.2)$ & $325(3.5)$ & 14.8 & $48(11.2)$ & $314(3.4)$ & 15.3 & $0(0.0)$ & $11(0.1)$ & $-^{\dagger \dagger}$ \\
\hline Florida & $786(24.3)$ & $7,062(3.2)$ & 11.1 & $759(23.5)$ & $6,906(3.1)$ & 11.0 & $27(0.8)$ & $156(0.1)$ & 17.3 \\
\hline Georgia & $421(22.8)$ & 4,394 (3.5) & 9.6 & $415(22.5)$ & $4,281(3.4)$ & 9.7 & $6(0.3)$ & $113(0.1)$ & 5.3 \\
\hline Hawaii & $151(35.5)$ & $529(3.1)$ & 28.5 & $142(33.4)$ & $511(3.0)$ & 27.8 & $9(2.1)$ & $18(0.1)$ & - $^{\dagger+}$ \\
\hline Idaho & $109(34.9)$ & $713(3.3)$ & 15.3 & 109 (34.9) & $701(3.3)$ & 15.5 & $0(0.0)$ & $12(0.1)$ & $-^{\dagger+}$ \\
\hline Illinois & $883(19.7)$ & $5,254(3.6)$ & 16.8 & $862(19.3)$ & $5,102(3.5)$ & 16.9 & $21(0.5)$ & $152(0.1)$ & 13.8 \\
\hline Indiana & $233(28.0)$ & $2,770(3.4)$ & 8.4 & $227(27.3)$ & $2,699(3.3)$ & 8.4 & $6(0.7)$ & $71(0.1)$ & 8.5 \\
\hline lowa & $146(21.2)$ & $1,352(3.6)$ & 10.8 & $140(20.3)$ & $1,320(3.5)$ & 10.6 & $6(0.9)$ & $32(0.1)$ & 18.8 \\
\hline Kansas & $68(17.0)$ & 1,164 (3.2) & 5.8 & $68(17.0)$ & $1,152(3.2)$ & 5.9 & $0(0.0)$ & $12(0.0)$ & $-^{\dagger \dagger}$ \\
\hline Kentucky & $164(30.8)$ & $1,831(3.4)$ & 9.0 & $153(28.7)$ & 1,799 (3.3) & 8.5 & $11(2.1)$ & $32(0.1)$ & 34.4 \\
\hline Louisiana & $109(20.2)$ & $2,089(3.5)$ & 5.2 & 一** & * $\quad 2,029(3.4)$ & 一** & —* & $60(0.1)$ & 一** \\
\hline Maine & $54(26.2)$ & $427(3.5)$ & 12.6 & $48(23.3)$ & $408(3.3)$ & 11.8 & $6(2.9)$ & $19(0.2)$ & $-^{\dagger+}$ \\
\hline Maryland & $352(16.1)$ & $2,488(3.5)$ & 14.1 & $340(15.5)$ & 2,397 (3.4) & 14.2 & $12(0.5)$ & $91(0.1)$ & 13.2 \\
\hline Massachusetts & $547(15.4)$ & $2,445(3.5)$ & 22.4 & $523(14.8)$ & $2,373(3.4)$ & 22.0 & $24(0.7)$ & $72(0.1)$ & 33.3 \\
\hline Michigan & $584(32.8)$ & 4,059 (3.7) & 14.4 & $560(31.5)$ & $3,950(3.6)$ & 14.2 & $24(1.3)$ & $109(0.1)$ & 22.0 \\
\hline Minnesota & $349(25.0)$ & $2,329(3.5)$ & 15.0 & $336(24.1)$ & $2,270(3.4)$ & 14.8 & $13(0.9)$ & $59(0.1)$ & 22.0 \\
\hline Mississippi & 48 (19.7) & 1,303 (3.5) & 3.7 & $42(17.2)$ & $1,251(3.4)$ & 3.4 & $6(2.5)$ & $52(0.1)$ & 11.5 \\
\hline Missouri & $298(30.8)$ & $2,658(3.6)$ & 11.2 & $285(29.4)$ & $2,593(3.5)$ & 11.0 & $13(1.3)$ & $65(0.1)$ & 20.0 \\
\hline Montana & $23(17.3)$ & $329(2.9)$ & 7.0 & 一** & 一* & 一* & - ${ }^{* *}$ & - ${ }^{* *}$ & 一** \\
\hline Nebraska & $108(31.1)$ & $937(3.7)$ & 11.5 & —** & 894 (3.5) & - ** & -** & $43(0.2)$ & 一** \\
\hline Nevada & $146(25.7)$ & $1,044(2.9)$ & 14.0 & $140(24.7)$ & $1,026(2.9)$ & 13.6 & $6(1.1)$ & $18(0.1)$ & - $^{\dagger+}$ \\
\hline New Hampshire & $110(25.1)$ & $416(3.5)$ & 26.4 & $110(25.1)$ & $416(3.5)$ & 26.4 & $0(0.0)$ & $0(0.0)$ & $-^{\dagger+}$ \\
\hline New Jersey & $614(14.5)$ & $3,556(3.5)$ & 17.3 & $599(14.1)$ & $3,457(3.4)$ & 17.3 & $15(0.4)$ & $99(0.1)$ & 15.2 \\
\hline New Mexico & $38(20.4)$ & $586(2.5)$ & 6.5 & 38 (20.4) & $573(2.5)$ & 6.6 & $0(0.0)$ & $13(0.1)$ & - $^{+\dagger}$ \\
\hline New York & $1,476(19.1)$ & $7,994(3.5)$ & 18.5 & $1,440(18.6)$ & $7,754(3.4)$ & 18.6 & $36(0.5)$ & $240(0.1)$ & 15.0 \\
\hline North Carolina & 419 (22.6) & $4,006(3.4)$ & 10.5 & $404(21.8)$ & $3,873(3.3)$ & 10.4 & $15(0.8)$ & $133(0.1)$ & 11.3 \\
\hline North Dakota & $43(32.3)$ & $346(3.3)$ & 12.4 & $43(32.3)$ & 一** & 一** & $0(0.0)$ & - $* *$ & - $* *$ \\
\hline Ohio & 357 (19.4) & $4,580(3.4)$ & 7.8 & 344 (18.7) & $4,431(3.3)$ & 7.8 & $13(0.7)$ & $149(0.1)$ & 8.7 \\
\hline Oklahoma & $122(31.0)$ & $1,671(3.4)$ & 7.3 & $122(31.0)$ & $1,642(3.3)$ & 7.4 & $0(0.0)$ & $29(0.1)$ & 0.0 \\
\hline Oregon & $222(29.4)$ & $1,490(3.5)$ & 14.9 & $210(27.8)$ & $1,464(3.5)$ & 14.3 & $12(1.6)$ & $26(0.1)$ & 46.2 \\
\hline Pennsylvania & 396 (15.6) & 4,474 (3.3) & 8.9 & $374(14.7)$ & $4,338(3.2)$ & 8.6 & $22(0.9)$ & $136(0.1)$ & 16.2 \\
\hline Puerto Rico & $38(45.2)$ & $447(2.1)$ & 8.5 & $38(45.2)$ & 一** & 一** & $0(0.0)$ & 一** & 一** \\
\hline Rhode Island & $61(20.3)$ & $383(3.6)$ & 15.9 & 一** & $365(3.5)$ & 一** & 一** & $18(0.2)$ & - **,+† \\
\hline South Carolina & $157(22.7)$ & $1,884(3.3)$ & 8.3 & $157(22.7)$ & $1,860(3.3)$ & 8.4 & $0(0.0)$ & $24(0.0)$ & 0.0 \\
\hline South Dakota & 38 (29.5) & $427(3.6)$ & 8.9 & 38 (29.5) & - ${ }^{* *}$ & 一** & $0(0.0)$ & -** & - ${ }^{* *}$ \\
\hline Tennessee & $193(24.3)$ & $2,709(3.4)$ & 7.1 & $181(22.8)$ & $2,624(3.2)$ & 6.9 & $12(1.5)$ & $85(0.1)$ & 14.1 \\
\hline Texas & $1,469(23.8)$ & $11,960(3.2)$ & 12.3 & $1,445(23.4)$ & $11,603(3.1)$ & 12.5 & $24(0.4)$ & $357(0.1)$ & 6.7 \\
\hline Utah & $305(28.0)$ & $1,629(3.5)$ & 18.7 & $299(27.4)$ & $1,571(3.3)$ & 19.0 & $6(0.5)$ & $58(0.1)$ & 10.3 \\
\hline Vermont & $29(24.2)$ & $183(3.4)$ & 15.8 & $29(24.2)$ & 一* & 一* & $0(0.0)$ & 一** & 一** \\
\hline Virginia & $393(16.8)$ & $3,473(3.5)$ & 11.3 & $381(16.3)$ & 3,368 (3.4) & 11.3 & $12(0.5)$ & $105(0.1)$ & 11.4 \\
\hline Washington & $325(18.8)$ & $2,579(3.0)$ & 12.6 & - ${ }^{* *}$ & * 2,501 (2.9) & - ${ }^{* *}$ & - ** & $78(0.1)$ & 一** \\
\hline West Virginia & $34(28.1)$ & $591(3.2)$ & 5.8 & $34(28.1)$ & 一** & 一** & $0(0.0)$ & —** & 一** \\
\hline Wisconsin & $252(27.5)$ & $2,303(3.6)$ & 10.9 & $240(26.2)$ & $2,236(3.5)$ & 10.7 & $12(1.3)$ & $67(0.1)$ & 17.9 \\
\hline Wyoming & $32(32.7)$ & $230(3.5)$ & 13.9 & $32(32.7)$ & $220(3.4)$ & 14.5 & $0(0.0)$ & $10(0.2)$ & - $^{\dagger+}$ \\
\hline Total & $16,001(21.4)$ & $127,508(3.3)$ & 12.5 & $15,532(20.7)$ & $123,974(3.3)$ & 12.5 & 469 (0.6) & $3,534(0.1)$ & 13.3 \\
\hline
\end{tabular}

See table footnotes on the next page. 
TABLE 4. (Continued) Number, percentage, and proportion of multiple-birth infants, twins, and triplets and higher-order infants born with use of assisted reproductive technology procedures, by female patient's area of residence at time of treatment — United States and Puerto Rico, 2018

Abbreviation: ART = assisted reproductive technology.

* In cases of missing patient's residence data $(0.3 \%)$, the patient's residence was assigned as the location where the ART procedure was performed.

t ART totals include infants conceived from ART procedures performed in 2017 and born in 2018 and infants conceived from ART procedures performed in 2018 and born in 2018. Total ART births exclude births to non-U.S. residents.

$\S$ Includes only the number of infants live born in a multiple-birth delivery. For example, if three infants were born in a live-birth delivery and one of the three infants was stillborn, the total number of live-born infants would be two. However, the two infants still would be counted as triplets.

" U.S. births exclude births to non-U.S. residents (Source: National Center for Health Statistics, Vital statistics data available. Natality public use file and CD-ROM. Hyattsville, MD, National Center for Health Statistics).

** To protect confidentiality, cells with values of 1-4 for ART infants and cells with values of $0-9$ for all infants are suppressed. Also suppressed are data that can be used to derive suppressed cell values. These values are included in the totals.

${ }^{+\dagger}$ Estimates on the basis of $\mathrm{N}<20$ in the denominator have been suppressed because such rates are considered unstable.

low birthweight among ART-conceived infants decreased from $20.2 \%$ to $18.3 \%$ (a $9.4 \%$ decrease), and preterm birth rates decreased from $27.8 \%$ to $26.1 \%$ (a $6.1 \%$ decrease). The contribution of ART-conceived twins to all twins born in the United States decreased from $14.7 \%$ to $12.5 \%$ (a $15.0 \%$ decrease). The contribution of ART-conceived infants to all triplets and higher-order infants decreased from $17.3 \%$ to $13.3 \%$ (a $23.1 \%$ decrease).

Despite these improvements, ART still disproportionally contributes to multiple births and poor birth outcomes (low birthweight and preterm birth). Because births resulting from ART are more likely to be multiple births than are births in the general population, and because multiple births are associated with higher rates of prematurity than singleton births, their contribution to poor birth outcomes continues to be noteworthy. In 2018, the multiple birth rate was nearly 6.5 times higher among ART-conceived infants compared with all infants $(21.4 \%$ versus $3.3 \%$ ). Although infants conceived with ART accounted for approximately $2.0 \%$ of total births in the United States in 2018, the proportion of multiple births attributable to ART was $12.5 \%$. The percentage of infants with low birthweight or born preterm was 2.2 and 2.6 times higher among ART-conceived infants (18.3\% and $26.1 \%$, respectively) than among all infants ( $8.3 \%$ and $10.0 \%$, respectively). Nationally, even among singletons, the rate of preterm birth among ART-conceived infants was 1.8 times the preterm birth rate among all infants (14.9\% and 8.3\%, respectively).

\section{Variations in ART Use by States and Territories}

The rate of ART use, as measured by number of procedures performed per 1 million women aged 15-44 years, increased from 3,040 in reporting year 2017 to 3,135 in reporting year 2018. ART use varied across areas. Residents of 14 states (California, Connecticut, Delaware, Hawaii, Illinois, Maryland, Massachusetts, New Hampshire, New Jersey, New York, Rhode Island, Utah, Vermont, and Virginia) and the District of Columbia had higher rates of ART use than the national rate. Residents of California, Illinois, Massachusetts,
New Jersey, New York, and Texas accounted for almost half (46.1\%) of all infants conceived with ART. The large number of ART procedures performed in these six states is a result of the size of the general population (California and Texas), high rates of ART use per capita (Illinois, Massachusetts, and New Jersey), or both (New York).

The contribution of ART to all infants born varied substantially by state. State-level differences might be explained in part by variations in insurance mandates for infertility treatments and disparities in access to fertility services (22-24). As of 2021, a total of 19 states (Arkansas, California, Colorado, Connecticut, Delaware, Hawaii, Illinois, Louisiana, Maryland, Massachusetts, Montana, New Hampshire, New Jersey, New York, Ohio, Rhode Island, Texas, Utah, and West Virginia) had passed fertility insurance coverage laws mandating that private insurers provide coverage for certain fertility treatments, although not all mandates require coverage for $\operatorname{ART}(25,26)$. Of four states that provide comprehensive coverage, two include at least four oocyte retrievals (Illinois and New Jersey), one does not limit the number of treatment cycles (Massachusetts), and one has a $\$ 100,000$ cap on treatment (Rhode Island). These four states had rates of ART use that were $\geq 50 \%$ higher than the national rate.

Other possible contributors to differences in ART use across states might include state variations in demographic characteristics such as race, ethnicity, and income levels. Use of fertility services varies by race and ethnicity. One study that analyzed 2014 NASS data indicated that ART use was highest among Asians or Pacific Islanders, followed by non-Hispanic White women, whereas non-Hispanic Black, Hispanic, and non-Hispanic American Indian or Alaska Native women had substantially lower levels of ART use (27). A study that linked NASS data to state vital statistics registries data in Florida, Massachusetts, and Michigan also found disparities by maternal race and ethnicity in ART use (24). Because many insurance plans in the United States do not provide coverage for ART treatment, costs often are the responsibility of patients (22). Even in states with an insurance mandate, ART use rates for non-Hispanic Black and Hispanic women were substantially 
TABLE 5. Number, percentage, and proportion of infants born with use of assisted reproductive technology, by low birthweight category and female patient's area of residence at time of treatment - United States and Puerto Rico, 2018

\begin{tabular}{|c|c|c|c|c|c|c|c|c|c|}
\hline \multirow{3}{*}{$\begin{array}{l}\text { Patient's area of } \\
\text { residence* }\end{array}$} & \multicolumn{3}{|c|}{$<1,500 \mathrm{~g}(\mathrm{VLBW})$} & \multicolumn{3}{|c|}{$1,500-2,499 \mathrm{~g}(\mathrm{MLBW})$} & \multicolumn{3}{|c|}{$<2,500 \mathrm{~g}(\mathrm{LBW})$} \\
\hline & ART infants $^{\dagger}$ & All infants ${ }^{\S}$ & $\begin{array}{c}\text { Proportion of ART } \\
\text { infants among } \\
\text { all infants } \\
\end{array}$ & ART infants $^{\dagger}$ & All infants ${ }^{\S}$ & $\begin{array}{c}\text { Proportion of ART } \\
\text { infants among } \\
\text { all infants } \\
\end{array}$ & ART infants ${ }^{\dagger}$ & All infants ${ }^{\S}$ & $\begin{array}{c}\text { Proportion of ART } \\
\text { infants among } \\
\text { all infants } \\
\end{array}$ \\
\hline & No. (\%) & No. (\%) & $\%$ & No. (\%) & No. (\%) & $\%$ & No. (\%) & No. (\%) & $\%$ \\
\hline Alabama & $21(5.7)$ & $1,084(1.9)$ & 1.9 & $52(14.1)$ & $5,100(8.8)$ & 1.0 & $73(19.8)$ & $6,184(10.7)$ & 1.2 \\
\hline Alaska & -9 & $98(1.0)$ & -9 & - & $492(4.9)$ & $-\pi$ & $15(20.0)$ & $590(5.8)$ & 2.5 \\
\hline Arizona & $45(3.5)$ & $977(1.2)$ & 4.6 & $278(21.8)$ & $5,139(6.4)$ & 5.4 & $323(25.3)$ & $6,116(7.6)$ & 5.3 \\
\hline Arkansas & $6(2.4)$ & $565(1.5)$ & 1.1 & $59(24.0)$ & $2,900(7.8)$ & 2.0 & $65(26.4)$ & $3,465(9.4)$ & 1.9 \\
\hline California & $212(2.2)$ & $5,039(1.1)$ & 4.2 & $1,378(14.2)$ & $26,706(5.9)$ & 5.2 & $1,590(16.4)$ & $31,745(7.0)$ & 5.0 \\
\hline Colorado & $26(1.9)$ & $761(1.2)$ & 3.4 & $260(19.2)$ & $5,145(8.2)$ & 5.1 & $286(21.1)$ & $5,906(9.4)$ & 4.8 \\
\hline Connecticut & $34(2.4)$ & 459 (1.3) & 7.4 & $208(14.7)$ & $2,182(6.3)$ & 9.5 & $242(17.1)$ & $2,641(7.6)$ & 9.2 \\
\hline Delaware & $8(3.6)$ & $161(1.5)$ & 5.0 & $21(9.5)$ & $787(7.4)$ & 2.7 & $29(13.1)$ & $948(8.9)$ & 3.1 \\
\hline District of Columbia & - & $165(1.8)$ & - & —? & $753(8.2)$ & $-\pi$ & $43(10.2)$ & $918(10.0)$ & 4.7 \\
\hline Florida & $137(4.3)$ & $3,482(1.6)$ & 3.9 & $527(16.7)$ & $15,735(7.1)$ & 3.3 & $664(21.0)$ & $19,217(8.7)$ & 3.5 \\
\hline Georgia & $46(2.6)$ & $2,259(1.8)$ & 2.0 & $306(17.5)$ & $10,474(8.3)$ & 2.9 & $352(20.1)$ & $12,733(10.1)$ & 2.8 \\
\hline Hawaii & $21(5.2)$ & $240(1.4)$ & 8.8 & $92(22.9)$ & $1,176(6.9)$ & 7.8 & $113(28.1)$ & $1,416(8.3)$ & 8.0 \\
\hline Idaho & $19(6.2)$ & $224(1.0)$ & 8.5 & $69(22.5)$ & $1,329(6.2)$ & 5.2 & $88(28.7)$ & $1,553(7.3)$ & 5.7 \\
\hline Illinois & $116(2.7)$ & $2,132(1.5)$ & 5.4 & $647(14.8)$ & $10,238(7.1)$ & 6.3 & $763(17.4)$ & $12,370(8.5)$ & 6.2 \\
\hline Indiana & 29 (3.6) & $1,111(1.4)$ & 2.6 & $156(19.3)$ & $5,461(6.7)$ & 2.9 & $185(22.9)$ & $6,572(8.0)$ & 2.8 \\
\hline lowa & $21(3.1)$ & $425(1.1)$ & 4.9 & $107(15.7)$ & $2,183(5.8)$ & 4.9 & $128(18.7)$ & $2,608(6.9)$ & 4.9 \\
\hline Kansas & $8(2.1)$ & 459 (1.3) & 1.7 & $55(14.7)$ & $2,217(6.1)$ & 2.5 & $63(16.8)$ & $2,676(7.4)$ & 2.4 \\
\hline Kentucky & $16(3.1)$ & $756(1.4)$ & 2.1 & 95 (18.4) & $4,026(7.5)$ & 2.4 & $111(21.6)$ & $4,782(8.9)$ & 2.3 \\
\hline Louisiana & $17(3.2)$ & $1,145(1.9)$ & 1.5 & $85(16.1)$ & $5,283(8.9)$ & 1.6 & 102 (19.4) & $6,428(10.8)$ & 1.6 \\
\hline Maine & -9 & $121(1.0)$ & - & - & $764(6.2)$ & - & $30(15.2)$ & $885(7.2)$ & 3.4 \\
\hline Maryland & $73(3.4)$ & $1,175(1.7)$ & 6.2 & $272(12.5)$ & $5,091(7.2)$ & 5.3 & 345 (15.9) & $6,266(8.8)$ & 5.5 \\
\hline Massachusetts & $71(2.1)$ & $822(1.2)$ & 8.6 & $454(13.1)$ & $4,415(6.4)$ & 10.3 & 525 (15.2) & $5,237(7.6)$ & 10.0 \\
\hline Michigan & 65 (3.8) & $1,485(1.3)$ & 4.4 & 325 (18.8) & $7,817(7.1)$ & 4.2 & $390(22.5)$ & $9,302(8.5)$ & 4.2 \\
\hline Minnesota & $51(3.7)$ & $786(1.2)$ & 6.5 & $227(16.4)$ & $3,831(5.7)$ & 5.9 & $278(20.1)$ & $4,617(6.9)$ & 6.0 \\
\hline Mississippi & $11(4.6)$ & $794(2.1)$ & 1.4 & 45 (18.9) & $3,690(10.0)$ & 1.2 & $56(23.5)$ & $4,484(12.1)$ & 1.2 \\
\hline Missouri & $28(3.1)$ & $991(1.4)$ & 2.8 & $184(20.1)$ & $5,398(7.4)$ & 3.4 & $212(23.2)$ & $6,389(8.7)$ & 3.3 \\
\hline Montana & - & $108(0.9)$ & - & - & $747(6.5)$ & $-\pi$ & $24(18.3)$ & 855 (7.4) & 2.8 \\
\hline Nebraska & $8(2.4)$ & $287(1.1)$ & 2.8 & $69(20.5)$ & $1,640(6.4)$ & 4.2 & $77(22.8)$ & $1,927(7.6)$ & 4.0 \\
\hline Nevada & $33(6.1)$ & 459 (1.3) & 7.2 & $102(19.0)$ & $2,638(7.4)$ & 3.9 & $135(25.1)$ & $3,097(8.7)$ & 4.4 \\
\hline New Hampshire & $12(2.8)$ & $131(1.1)$ & 9.2 & $67(15.5)$ & $681(5.7)$ & 9.8 & $79(18.2)$ & $812(6.8)$ & 9.7 \\
\hline New Jersey & $102(2.5)$ & $1,401(1.4)$ & 7.3 & $512(12.3)$ & $6,600(6.5)$ & 7.8 & $614(14.8)$ & 8,001 (7.9) & 7.7 \\
\hline New Mexico & — & 294 (1.3) & - & - & $1,790(7.8)$ & -9 & 48 (25.9) & $2,084(9.0)$ & 2.3 \\
\hline New York & $212(2.9)$ & $2,968(1.3)$ & 7.1 & $1,001(13.7)$ & $15,240(6.7)$ & 6.6 & $1,213(16.6)$ & $18,208(8.0)$ & 6.7 \\
\hline North Carolina & $63(3.7)$ & $1,887(1.6)$ & 3.3 & $250(14.7)$ & $9,083(7.6)$ & 2.8 & $313(18.4)$ & $10,970(9.2)$ & 2.9 \\
\hline North Dakota & $11(8.7)$ & $121(1.1)$ & 9.1 & $22(17.5)$ & $577(5.4)$ & 3.8 & $33(26.2)$ & $698(6.6)$ & 4.7 \\
\hline Ohio & $47(2.7)$ & $1,995(1.5)$ & 2.4 & $253(14.5)$ & $9,476(7.0)$ & 2.7 & $300(17.2)$ & $11,471(8.5)$ & 2.6 \\
\hline Oklahoma & $11(2.8)$ & $721(1.4)$ & 1.5 & $81(20.8)$ & $3,394(6.8)$ & 2.4 & $92(23.7)$ & $4,115(8.3)$ & 2.2 \\
\hline Oregon & $31(4.2)$ & $370(0.9)$ & 8.4 & $120(16.3)$ & $2,456(5.8)$ & 4.9 & $151(20.5)$ & $2,826(6.7)$ & 5.3 \\
\hline Pennsylvania & $66(2.7)$ & $1,869(1.4)$ & 3.5 & $308(12.7)$ & $9,353(6.9)$ & 3.3 & $374(15.4)$ & $11,222(8.3)$ & 3.3 \\
\hline Puerto Rico & $9(10.7)$ & $302(1.4)$ & 3.0 & $26(31.0)$ & $1,910(8.9)$ & 1.4 & $35(41.7)$ & $2,212(10.3)$ & 1.6 \\
\hline Rhode Island & $15(5.2)$ & $136(1.3)$ & 11.0 & 41 (14.2) & $665(6.3)$ & 6.2 & $56(19.4)$ & $801(7.6)$ & 7.0 \\
\hline South Carolina & $20(3.1)$ & $965(1.7)$ & 2.1 & $112(17.3)$ & 4,467 (7.9) & 2.5 & $132(20.3)$ & $5,432(9.6)$ & 2.4 \\
\hline South Dakota & $10(7.9)$ & $104(0.9)$ & 9.6 & $22(17.5)$ & $685(5.8)$ & 3.2 & $32(25.4)$ & 789 (6.6) & 4.1 \\
\hline Tennessee & $34(4.4)$ & $1,303(1.6)$ & 2.6 & $126(16.5)$ & $6,168(7.6)$ & 2.0 & $160(20.9)$ & 7,471 (9.3) & 2.1 \\
\hline Texas & $211(3.5)$ & $5,345(1.4)$ & 3.9 & $1,021(17.1)$ & $26,692(7.0)$ & 3.8 & $1,232(20.6)$ & $32,037(8.5)$ & 3.8 \\
\hline Utah & $44(4.2)$ & $495(1.0)$ & 8.9 & $190(18.1)$ & $2,890(6.1)$ & 6.6 & $234(22.2)$ & $3,385(7.2)$ & 6.9 \\
\hline Vermont & - & $58(1.1)$ & -9 & - & $323(5.9)$ & $-\pi$ & 17 (14.9) & $381(7.0)$ & 4.5 \\
\hline Virginia & $67(2.9)$ & $1,453(1.5)$ & 4.6 & $275(12.1)$ & $6,722(6.7)$ & 4.1 & $342(15.0)$ & $8,175(8.2)$ & 4.2 \\
\hline Washington & $30(1.8)$ & $865(1.0)$ & 3.5 & $261(15.3)$ & $4,825(5.6)$ & 5.4 & $291(17.1)$ & $5,690(6.6)$ & 5.1 \\
\hline West Virginia & - & 285 (1.6) & - & — & $1,423(7.8)$ & - & 24 (20.9) & $1,708(9.4)$ & 1.4 \\
\hline Wisconsin & $19(2.1)$ & 765 (1.2) & 2.5 & $158(17.8)$ & $4,170(6.5)$ & 3.8 & 177 (19.9) & 4,935 (7.7) & 3.6 \\
\hline Wyoming & - & $70(1.1)$ & -9 & - & $544(8.3)$ & -9 & $25(25.8)$ & $614(9.4)$ & 4.1 \\
\hline Total & $2,158(3.0)$ & $52,473(1.4)$ & 4.1 & $11,123(15.4)$ & $263,491(6.9)$ & 4.2 & $13,281(18.3)$ & $315,964(8.3)$ & 4.2 \\
\hline
\end{tabular}

Abbreviations: ART = assisted reproductive technology; LBW = low birthweight; MLBW = moderately low birthweight; VLBW = very low birthweight.

* In cases of missing patient's residence data $(0.3 \%)$, the patient's residence was assigned as the location where the ART procedure was performed.

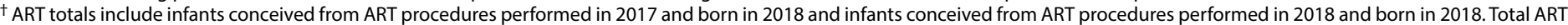
infants exclude births to non-U.S. residents and include only infants with birthweight data available.

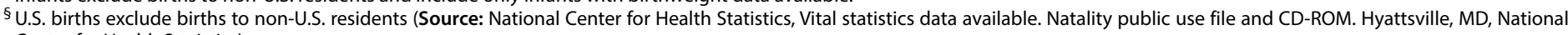
Center for Health Statistics).

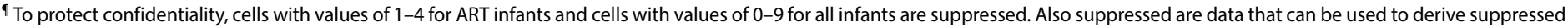
cell values. These values are included in the totals. 
TABLE 6. Number, percentage, and proportion of infants born with use of assisted reproductive technology, by preterm gestational age category and female patient's area of residence at time of treatment - United States and Puerto Rico, 2018

\begin{tabular}{|c|c|c|c|c|c|c|c|c|c|c|c|c|}
\hline \multirow{3}{*}{$\begin{array}{l}\text { Patient's area of } \\
\text { residence* }\end{array}$} & \multicolumn{3}{|c|}{ VPTB (<32 wks) } & \multicolumn{3}{|c|}{ Early PTB (<34 wks) } & \multicolumn{3}{|c|}{ Late PTB (34-36 wks) } & \multicolumn{3}{|c|}{ PTB (<37 wks) } \\
\hline & $\begin{array}{c}\text { ART } \\
\text { infants }^{\dagger} \\
\end{array}$ & All infants & $\begin{array}{l}\text { Proportion } \\
\text { of ART } \\
\text { infants } \\
\text { among all } \\
\text { infants }\end{array}$ & ART infants ${ }^{\dagger}$ & All infants ${ }^{\S}$ & $\begin{array}{c}\text { Proportion } \\
\text { of ART } \\
\text { infants } \\
\text { among all } \\
\text { infants }\end{array}$ & $\mathrm{ART}_{\text {infants }}^{\dagger}$ & All infants $§$ & $\begin{array}{c}\text { Proportion } \\
\text { of ART } \\
\text { infants } \\
\text { among all } \\
\text { infants }\end{array}$ & ART infants ${ }^{\dagger}$ & All infants ${ }^{\S}$ & $\begin{array}{c}\text { Proportion } \\
\text { of ART } \\
\text { infants } \\
\text { among all } \\
\text { infants }\end{array}$ \\
\hline & No. (\%) & No. (\%) & $\%$ & No. (\%) & No. (\%) & $\%$ & No. (\%) & No. (\%) & $\%$ & No. (\%) & No. (\%) & $\%$ \\
\hline Alabama & $32(8.3)$ & $1,194(2.1)$ & 2.7 & 46 (11.9) & 2,088 (3.6) & 2.2 & 72 (18.6) & $5,116(8.9)$ & 1.4 & $118(30.5)$ & $7,204(12.5)$ & 1.6 \\
\hline Alaska & - & $131(1.3)$ & 0.8 & - & $236(2.3)$ & - & — & 697 (6.9) & - & $12(16.4)$ & $933(9.3)$ & 1.3 \\
\hline Arizona & $67(5.0)$ & $1,127(1.4)$ & 5.9 & $135(10.2)$ & $1,968(2.4)$ & 6.9 & $330(24.8)$ & $5,705(7.1)$ & 5.8 & $465(35.0)$ & $7,673(9.5)$ & 6.1 \\
\hline Arkansas & $10(4.1)$ & 644 (1.7) & 1.6 & $15(6.1)$ & $1,154(3.1)$ & 1.3 & $64(26.1)$ & $3,145(8.5)$ & 2.0 & 79 (32.2) & 4,299 (11.6) & 1.8 \\
\hline California & $324(3.3)$ & $5,888(1.3)$ & 5.5 & $667(6.7)$ & $10,438(2.3)$ & 6.4 & $1,620(16.3)$ & $29,526(6.5)$ & 5.5 & $2,287(23.0)$ & $39,964(8.8)$ & 5.7 \\
\hline Colorado & $40(2.8)$ & $887(1.4)$ & 4.5 & $100(7.1)$ & $1,551(2.5)$ & 6.4 & 305 (21.7) & $4,244(6.7)$ & 7.2 & 405 (28.8) & $5,795(9.2)$ & 7.0 \\
\hline Connecticut & $40(2.8)$ & $517(1.5)$ & 7.7 & $112(7.9)$ & 905 (2.6) & 12.4 & $213(15.0)$ & $2,346(6.8)$ & 9.1 & 325 (22.9) & $3,251(9.4)$ & 10.0 \\
\hline Delaware & $12(5.3)$ & $174(1.6)$ & 6.9 & $14(6.1)$ & $293(2.8)$ & 4.8 & 29 (12.7) & 722 (6.8) & 4.0 & 43 (18.9) & $1,015(9.6)$ & 4.2 \\
\hline District of Columbia & $-9(0.5)$ & $181(2.0)$ & 1.1 & $8(1.9)$ & $311(3.4)$ & 2.6 & $60(14.1)$ & $619(6.7)$ & 9.7 & $68(15.9)$ & $930(10.1)$ & 7.3 \\
\hline Florida & $180(5.6)$ & $3,804(1.7)$ & 4.7 & $318(9.9)$ & $6,457(2.9)$ & 4.9 & 673 (20.9) & $16,244(7.3)$ & 4.1 & $991(30.8)$ & $22,701(10.2)$ & 4.4 \\
\hline Georgia & $67(3.7)$ & $2,539(2.0)$ & 2.6 & $125(6.8)$ & $4,340(3.4)$ & 2.9 & $416(22.8)$ & $10,169(8.1)$ & 4.1 & $541(29.6)$ & $14,509(11.5)$ & 3.7 \\
\hline Hawaii & $29(6.8)$ & $270(1.6)$ & 10.7 & $48(11.3)$ & $459(2.7)$ & 10.5 & $85(20.0)$ & $1,285(7.6)$ & 6.6 & $133(31.3)$ & $1,744(10.3)$ & 7.6 \\
\hline Idaho & $27(8.7)$ & $265(1.2)$ & 10.2 & $50(16.0)$ & $463(2.2)$ & 10.8 & $71(22.8)$ & $1,470(6.9)$ & 4.8 & $121(38.8)$ & $1,933(9.0)$ & 6.3 \\
\hline Illinois & $156(3.5)$ & $2,401(1.7)$ & 6.5 & $330(7.4)$ & $4,292(3.0)$ & 7.7 & $811(18.2)$ & $11,153(7.7)$ & 7.3 & $1,141(25.6)$ & $15,445(10.7)$ & 7.4 \\
\hline Indiana & $43(5.2)$ & $1,307(1.6)$ & 3.3 & $73(8.8)$ & $2,254(2.8)$ & 3.2 & $199(24.0)$ & $6,097(7.5)$ & 3.3 & $272(32.8)$ & $8,351(10.2)$ & 3.3 \\
\hline lowa & $31(4.5)$ & $530(1.4)$ & 5.8 & $70(10.2)$ & $993(2.6)$ & 7.0 & $140(20.3)$ & $2,752(7.3)$ & 5.1 & $210(30.5)$ & $3,745(9.9)$ & 5.6 \\
\hline Kansas & $12(3.0)$ & $511(1.4)$ & 2.3 & $29(7.2)$ & $922(2.5)$ & 3.1 & $70(17.5)$ & $2,518(6.9)$ & 2.8 & 99 (24.7) & $3,440(9.5)$ & 2.9 \\
\hline Kentucky & $21(4.0)$ & 878 (1.6) & 2.4 & $54(10.2)$ & $1,607(3.0)$ & 3.4 & 105 (19.8) & $4,502(8.3)$ & 2.3 & $159(30.0)$ & 6,109 (11.3) & 2.6 \\
\hline Louisiana & $29(5.4)$ & $1,293(2.2)$ & 2.2 & $50(9.3)$ & $2,167(3.6)$ & 2.3 & $119(22.2)$ & $5,576(9.4)$ & 2.1 & 169 (31.6) & $7,743(13.0)$ & 2.2 \\
\hline Maine & $7(3.4)$ & $140(1.1)$ & 5.0 & $15(7.3)$ & $266(2.2)$ & 5.6 & 36 (17.6) & $788(6.4)$ & 4.6 & 51 (24.9) & 1,054 (8.6) & 4.8 \\
\hline Maryland & $85(3.9)$ & $1,308(1.8)$ & 6.5 & $159(7.3)$ & $2,189(3.1)$ & 7.3 & $342(15.6)$ & $5,042(7.1)$ & 6.8 & $501(22.9)$ & $7,231(10.2)$ & 6.9 \\
\hline Massachusetts & $85(2.4)$ & $921(1.3)$ & 9.2 & 199 (5.6) & $1,682(2.4)$ & 11.8 & $556(15.7)$ & $4,490(6.5)$ & 12.4 & $755(21.4)$ & $6,172(8.9)$ & 12.2 \\
\hline Michigan & $76(4.3)$ & $1,686(1.5)$ & 4.5 & $176(9.9)$ & $2,973(2.7)$ & 5.9 & 351 (19.8) & $8,066(7.3)$ & 4.4 & $527(29.8)$ & $11,039(10.0)$ & 4.8 \\
\hline Minnesota & $70(5.0)$ & 890 (1.3) & 7.9 & $125(9.0)$ & $1,551(2.3)$ & 8.1 & $246(17.6)$ & $4,453(6.6)$ & 5.5 & $371(26.6)$ & $6,004(8.9)$ & 6.2 \\
\hline Mississippi & $19(7.8)$ & $888(2.4)$ & 2.1 & $32(13.2)$ & $1,536(4.2)$ & 2.1 & 45 (18.5) & $3,733(10.1)$ & 1.2 & 77 (31.7) & $5,269(14.2)$ & 1.5 \\
\hline Missouri & $33(3.4)$ & $1,184(1.6)$ & 2.8 & 85 (8.8) & $2,072(2.8)$ & 4.1 & $216(22.5)$ & $5,777(7.9)$ & 3.7 & 301 (31.3) & $7,849(10.7)$ & 3.8 \\
\hline Montana & - & $123(1.1)$ & 3.3 & $14(10.5)$ & $257(2.2)$ & 5.4 & 19 (14.3) & 790 (6.9) & 2.4 & $33(24.8)$ & $1,047(9.1)$ & 3.2 \\
\hline Nebraska & $9(2.6)$ & $370(1.5)$ & 2.4 & $27(7.8)$ & $665(2.6)$ & 4.1 & $111(32.0)$ & $1,999(7.8)$ & 5.6 & $138(39.8)$ & $2,664(10.5)$ & 5.2 \\
\hline Nevada & $41(7.3)$ & $532(1.5)$ & 7.7 & $64(11.4)$ & $962(2.7)$ & 6.7 & $102(18.1)$ & $2,654(7.4)$ & 3.8 & $166(29.5)$ & $3,616(10.1)$ & 4.6 \\
\hline New Hampshire & $11(2.5)$ & $152(1.3)$ & 7.2 & $30(6.9)$ & $276(2.3)$ & 10.9 & 68 (15.6) & $719(6.0)$ & 9.5 & $98(22.4)$ & $995(8.3)$ & 9.8 \\
\hline New Jersey & $136(3.2)$ & $1,518(1.5)$ & 9.0 & $258(6.1)$ & $2,655(2.6)$ & 9.7 & $660(15.6)$ & $6,963(6.9)$ & 9.5 & $918(21.8)$ & $9,618(9.5)$ & 9.5 \\
\hline New Mexico & $-1(2.2)$ & $327(1.4)$ & 1.2 & $12(6.5)$ & $580(2.5)$ & 2.1 & 49 (26.5) & $1,674(7.3)$ & 2.9 & $61(33.0)$ & $2,254(9.8)$ & 2.7 \\
\hline New York & $270(3.5)$ & $3,266(1.4)$ & 8.3 & $494(6.4)$ & $5,799(2.6)$ & 8.5 & $1,206(15.7)$ & $14,482(6.4)$ & 8.3 & $1,700(22.1)$ & $20,281(9.0)$ & 8.4 \\
\hline North Carolina & 77 (4.2) & 2,095 (1.8) & 3.7 & $157(8.5)$ & $3,581(3.0)$ & 4.4 & 364 (19.7) & $8,759(7.4)$ & 4.2 & $521(28.2)$ & $12,340(10.4)$ & 4.2 \\
\hline North Dakota & $11(8.3)$ & $128(1.2)$ & 8.6 & $18(13.6)$ & $262(2.5)$ & 6.9 & $32(24.2)$ & 756 (7.1) & 4.2 & 50 (37.9) & $1,018(9.6)$ & 4.9 \\
\hline Ohio & $66(3.6)$ & $2,262(1.7)$ & 2.9 & 139 (7.6) & $3,896(2.9)$ & 3.6 & $300(16.3)$ & 9,949 (7.4) & 3.0 & 439 (23.9) & $13,845(10.2)$ & 3.2 \\
\hline Oklahoma & $18(4.6)$ & $823(1.7)$ & 2.2 & $38(9.7)$ & $1,478(3.0)$ & 2.6 & $93(23.7)$ & $4,192(8.4)$ & 2.2 & $131(33.4)$ & $5,670(11.4)$ & 2.3 \\
\hline Oregon & $51(6.8)$ & $437(1.0)$ & 11.7 & $81(10.7)$ & 813 (1.9) & 10.0 & $119(15.8)$ & 2,491 (5.9) & 4.8 & $200(26.5)$ & $3,304(7.8)$ & 6.1 \\
\hline Pennsylvania & $85(3.4)$ & $2,124(1.6)$ & 4.0 & $161(6.4)$ & $3,688(2.7)$ & 4.4 & $446(17.6)$ & $9,227(6.8)$ & 4.8 & $607(24.0)$ & $12,915(9.5)$ & 4.7 \\
\hline Puerto Rico & $9(10.7)$ & $459(2.1)$ & 2.0 & $15(17.9)$ & $863(4.0)$ & 1.7 & $26(31.0)$ & $2,235(10.4)$ & 1.2 & $41(48.8)$ & $3,098(14.5)$ & 1.3 \\
\hline Rhode Island & $23(7.6)$ & $143(1.4)$ & 16.1 & $29(9.6)$ & $276(2.6)$ & 10.5 & $48(15.9)$ & $667(6.3)$ & 7.2 & 77 (25.6) & $943(9.0)$ & 8.2 \\
\hline South Carolina & 25 (3.6) & 1,083 (1.9) & 2.3 & $61(8.9)$ & 1,855 (3.3) & 3.3 & $143(20.8)$ & $4,571(8.1)$ & 3.1 & 204 (29.6) & $6,426(11.3)$ & 3.2 \\
\hline South Dakota & $8(6.3)$ & $141(1.2)$ & 5.7 & $17(13.3)$ & $254(2.1)$ & 6.7 & $22(17.2)$ & 868 (7.3) & 2.5 & $39(30.5)$ & $1,122(9.4)$ & 3.5 \\
\hline Tennessee & $46(5.8)$ & $1,441(1.8)$ & 3.2 & $84(10.6)$ & $2,531(3.1)$ & 3.3 & $153(19.3)$ & 6,380 (7.9) & 2.4 & 237 (29.9) & $8,911(11.0)$ & 2.7 \\
\hline Texas & $335(5.4)$ & $6,182(1.6)$ & 5.4 & $560(9.1)$ & $10,983(2.9)$ & 5.1 & $1,375(22.4)$ & $29,816(7.9)$ & 4.6 & $1,935(31.5)$ & $40,799(10.8)$ & 4.7 \\
\hline Utah & $52(4.8)$ & $600(1.3)$ & 8.7 & $101(9.3)$ & $1,053(2.2)$ & 9.6 & $260(24.0)$ & $3,392(7.2)$ & 7.7 & $361(33.3)$ & $4,445(9.4)$ & 8.1 \\
\hline Vermont & $-9(2.5)$ & $66(1.2)$ & 4.5 & $12(10.1)$ & $116(2.1)$ & 10.3 & 20 (16.8) & $346(6.4)$ & 5.8 & 32 (26.9) & $462(8.5)$ & 6.9 \\
\hline Virginia & $100(4.3)$ & $1,613(1.6)$ & 6.2 & $178(7.6)$ & $2,643(2.6)$ & 6.7 & $366(15.7)$ & $6,758(6.8)$ & 5.4 & $544(23.3)$ & $9,401(9.4)$ & 5.8 \\
\hline Washington & 49 (2.8) & $1,020(1.2)$ & 4.8 & $116(6.7)$ & $1,854(2.2)$ & 6.3 & $284(16.4)$ & $5,293(6.1)$ & 5.4 & $400(23.1)$ & $7,147(8.3)$ & 5.6 \\
\hline West Virginia & - $(2.5)$ & $311(1.7)$ & 1.0 & $9(7.5)$ & $566(3.1)$ & 1.6 & $27(22.5)$ & $1,592(8.7)$ & 1.7 & $36(30.0)$ & $2,158(11.8)$ & 1.7 \\
\hline Wisconsin & $32(3.5)$ & 924 (1.4) & 3.5 & $82(8.9)$ & $1,664(2.6)$ & 4.9 & $206(22.5)$ & $4,684(7.3)$ & 4.4 & $288(31.4)$ & $6,348(9.9)$ & 4.5 \\
\hline Wyoming & - $(2.0)$ & $92(1.4)$ & 2.2 & $5(5.1)$ & $157(2.4)$ & 3.2 & 29 (29.6) & $489(7.5)$ & 5.9 & $34(34.7)$ & $646(9.8)$ & 5.3 \\
\hline Total & $2,968(4.0)$ & $59,790(1.6)$ & 5.0 & $5,805(7.8)$ & $104,894(2.8)$ & 5.5 & $13,706(18.4)$ & $277,981(7.3)$ & 4.9 & $19,511(26.1)$ & $382,875(10.0)$ & 5.1 \\
\hline
\end{tabular}

Abbreviations: ART = assisted reproductive technology; PTB = preterm birth; VPTB = very preterm birth.

* In cases of missing patient's residence data $(0.3 \%)$, the patient's residence was assigned as the location where the ART procedure was performed.

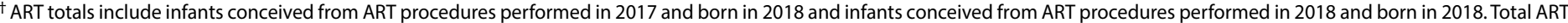
births exclude births to non-U.S. residents and include only infants with gestational age data available.

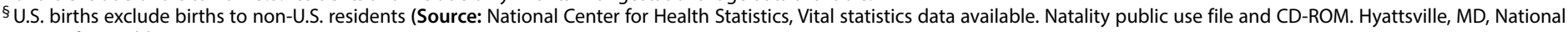
Center for Health Statistics).

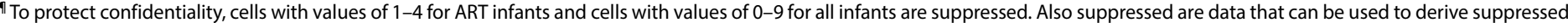
cell values. These values are included in the totals. 
TABLE 7. Percentage of low birthweight, preterm, and small for gestational age infants among singleton infants born with assisted reproductive technology, by female patient's area of residence at time of treatment - United States and Puerto Rico, 2018

\begin{tabular}{|c|c|c|c|c|c|c|}
\hline \multirow[b]{2}{*}{ Patient's area of residence ${ }^{*}$} & \multicolumn{2}{|c|}{$\begin{array}{l}\text { Low birthweight } \\
(<2,500 \mathrm{~g})\end{array}$} & \multicolumn{2}{|c|}{$\begin{array}{l}\text { Preterm } \\
(<37 \text { wks })\end{array}$} & \multicolumn{2}{|c|}{$\begin{array}{l}\text { Small for gestational age } \\
(22-44 \text { wks })\end{array}$} \\
\hline & $\begin{array}{l}\text { ART infants }{ }^{\dagger} \\
(\%)\end{array}$ & $\begin{array}{c}\text { All infants } \\
(\%)\end{array}$ & $\begin{array}{l}\text { ART infants }{ }^{\dagger} \\
(\%)\end{array}$ & $\begin{array}{l}\text { All infants } \\
(\%)\end{array}$ & $\begin{array}{l}\text { ART infants } \\
(\%)\end{array}$ & $\begin{array}{c}\text { All infants } \\
(\%)\end{array}$ \\
\hline Alabama & 8.2 & 8.7 & 15.2 & 10.4 & 6.9 & 11.6 \\
\hline Alaska & -9 & 4.4 & 10.9 & 7.5 & - & 5.7 \\
\hline Arizona & 10.3 & 5.9 & 17.2 & 7.7 & 6.7 & 8.7 \\
\hline Arkansas & 8.7 & 7.6 & 13.5 & 9.8 & 8.1 & 9.9 \\
\hline California & 7.5 & 5.5 & 12.8 & 7.2 & 7.8 & 8.7 \\
\hline Colorado & 9.0 & 7.6 & 15.1 & 7.5 & 9.9 & 12.8 \\
\hline Connecticut & 8.1 & 5.9 & 12.8 & 7.6 & 7.4 & 8.4 \\
\hline Delaware & 9.6 & 7.6 & 16.4 & 8.2 & 6.8 & 10.2 \\
\hline District of Columbia & 7.2 & 8.4 & 11.6 & 8.4 & 8.4 & 11.5 \\
\hline Florida & 8.0 & 7.1 & 17.4 & 8.6 & 6.7 & 9.9 \\
\hline Georgia & 8.2 & 8.2 & 15.8 & 9.5 & 6.8 & 11.3 \\
\hline Hawaii & 14.3 & 6.9 & 18.2 & 8.7 & 12.6 & 10.4 \\
\hline Idaho & 9.5 & 5.6 & 18.7 & 7.2 & 6.0 & 8.8 \\
\hline Illinois & 8.6 & 6.8 & 16.2 & 8.8 & 7.3 & 9.0 \\
\hline Indiana & 8.4 & 6.4 & 16.4 & 8.4 & 4.6 & 8.7 \\
\hline lowa & 8.2 & 5.3 & 18.1 & 8.0 & 5.2 & 6.8 \\
\hline Kansas & 6.5 & 5.8 & 13.5 & 7.7 & 5.8 & 7.8 \\
\hline Kentucky & 6.7 & 7.1 & 13.1 & 9.4 & 6.7 & 8.7 \\
\hline Louisiana & 8.6 & 8.9 & 17.4 & 10.9 & 5.0 & 10.9 \\
\hline Maine & 5.4 & 5.7 & 11.3 & 7.0 & 5.4 & 8.2 \\
\hline Maryland & 9.0 & 7.1 & 15.6 & 8.3 & 6.8 & 9.6 \\
\hline Massachusetts & 8.4 & 5.8 & 13.1 & 7.0 & 8.3 & 8.9 \\
\hline Michigan & 9.9 & 6.7 & 14.6 & 8.1 & 7.9 & 9.5 \\
\hline Minnesota & 8.0 & 5.3 & 13.2 & 7.2 & 6.7 & 7.4 \\
\hline Mississippi & 13.7 & 10.1 & 19.0 & 12.1 & 5.3 & 12.0 \\
\hline Missouri & 8.2 & 6.9 & 16.3 & 8.6 & 7.4 & 9.0 \\
\hline Montana & 7.3 & 6.0 & 12.7 & 7.5 & 9.1 & 9.3 \\
\hline Nebraska & 8.9 & 5.8 & 20.5 & 8.4 & 8.1 & 7.4 \\
\hline Nevada & 11.2 & 7.1 & 16.4 & 8.6 & 8.7 & 10.8 \\
\hline New Hampshire & 5.9 & 5.2 & 11.0 & 6.6 & 6.5 & 7.8 \\
\hline New Jersey & 7.7 & 6.1 & 14.2 & 7.7 & 7.4 & 9.5 \\
\hline New Mexico & 15.0 & 7.7 & 21.1 & 8.3 & 13.7 & 12.2 \\
\hline New York & 8.0 & 6.3 & 13.2 & 7.2 & 8.1 & 10.0 \\
\hline North Carolina & 7.9 & 7.5 & 14.8 & 8.5 & 7.5 & 10.2 \\
\hline North Dakota & - & 4.9 & 12.4 & 7.6 & - & 6.3 \\
\hline Ohio & 8.1 & 6.8 & 13.8 & 8.4 & 7.2 & 9.3 \\
\hline Oklahoma & 7.1 & 6.6 & 14.4 & 9.4 & 5.2 & 8.3 \\
\hline Oregon & 5.9 & 5.1 & 12.7 & 6.3 & 5.7 & 8.1 \\
\hline Pennsylvania & 8.1 & 6.7 & 15.3 & 7.9 & 5.7 & 9.6 \\
\hline Puerto Rico & 21.7 & 9.2 & 32.6 & 13.4 & 13.0 & 13.5 \\
\hline Rhode Island & 6.5 & 5.8 & 12.5 & 7.1 & 9.1 & 8.7 \\
\hline South Carolina & 8.8 & 7.9 & 16.7 & 9.5 & 6.2 & 10.1 \\
\hline South Dakota & 10.2 & 5.0 & 18.9 & 7.5 & - & 7.6 \\
\hline Tennessee & 9.9 & 7.5 & 17.7 & 9.2 & 5.7 & 10.0 \\
\hline Texas & 9.6 & 6.8 & 18.1 & 9.0 & 6.4 & 9.2 \\
\hline Utah & 12.0 & 5.3 & 17.5 & 7.4 & 9.8 & 8.1 \\
\hline Vermont & 8.0 & 5.6 & 17.8 & 7.0 & 9.3 & 8.0 \\
\hline Virginia & 7.5 & 6.5 & 14.7 & 7.7 & 6.5 & 9.5 \\
\hline Washington & 8.2 & 5.3 & 13.6 & 6.9 & 6.4 & 7.8 \\
\hline West Virginia & 9.6 & 7.6 & 18.6 & 9.9 & 7.2 & 9.7 \\
\hline Wisconsin & 6.7 & 5.9 & 15.0 & 7.9 & 6.1 & 8.0 \\
\hline Wyoming & 12.3 & 7.5 & 18.2 & 8.0 & 9.2 & 13.0 \\
\hline Total & 8.3 & 6.6 & 14.9 & 8.3 & 7.3 & 9.4 \\
\hline
\end{tabular}

Abbreviation: ART = assisted reproductive technology.

* In cases of missing patient's residence data $(0.3 \%)$, the patient's residence was assigned as the location where the ART procedure was performed.

+ ART totals include infants conceived from ART procedures performed in 2017 and born in 2018 and infants conceived from ART procedures performed in 2018 and born in 2018. Total ART births exclude births to non- U.S. residents and include only infants with gestational age data available.

$\S$ U.S. births exclude births to non-U.S. residents (Source: National Center for Health Statistics, Vital statistics data available. Natality public use file and CD-ROM. Hyattsville, MD, National Center for Health Statistics).

"To protect confidentiality, cells with values of 1-4 for ART infants and cells with values of $0-9$ for all infants are suppressed. Also suppressed are data that can be used to derive suppressed cell values. These values are included in the totals. 
lower than the rate for non-Hispanic White women (27). Thus, differences in the use rate might be due to factors beyond costs (27). Disparities might be due to cultural and social barriers, such as stigma, which might delay or prevent certain racial and ethnic groups from seeking infertility care (28).

Limitations of current data collection systems in terms of data quality and completeness might limit meaningful research in racial and ethnic disparities in ART use and outcomes (27). As of January 2016, all states had adopted the 2003 revision of the birth certificate that includes information on whether the pregnancy resulted from the use of infertility treatment; 47 states and the District of Columbia differentiate between the use of ART and non-ART treatments. This additional information on ART use in birth certificates could facilitate more comprehensive research on racial and ethnic differences in ART use and outcomes among infants born in the United States. The scope of such research would be greater if data from NASS, which include detailed information on ART, were to be linked to data from state vital statistics registries.

\section{Single-Embryo Transfer Rates}

Recommendations issued by the American Society for Reproductive Medicine and SART to limit the number of embryos to transfer have been revised multiple times to reduce higher-order multiple deliveries (29-32). The most recent guidance was intended to reduce all multiple births, including twins (3). This guidance adopted a broader approach and recommended SET for patients of any age transferring a euploid embryo, selected with the assistance of preimplantation genetic screening, and for patients aged $<38$ years with any one of the following criteria: 1) availability of quality embryos for cryopreservation, 2) a history of live birth after an IVF procedure, 3) availability of vitrified blastocyst stage embryos, or 4) undergoing first frozen-embryo transfer (3). Increasing SET rates among all age groups reflect these changes in clinical practice. However, variations in the percentages of SET procedures across states and territories suggest that SET has not been implemented equally in all areas.

\section{ART Multiple Births}

Singleton births have lower risks than multiple births for adverse outcomes, such as prematurity, low birthweight, developmental disability, and death $(10,33,34)$. To optimize healthy birth outcomes, the transfer of fewer embryos should be encouraged when clinically appropriate, taking into consideration the patient's age and prognosis $(3,35)$. The percentage of multiple births among ART-conceived infants in the United States has decreased from 53.1\% in 2000 (when national multiple birth rates were first reported) to $21.4 \%$ in 2018 (36). A substantial decrease was noted for both the percentage of ART-conceived triplets and higher-order multiples (from $8.9 \%$ in 2000 to $0.6 \%$ in 2018 ) and the percentage of ART-conceived twins (from $44.2 \%$ in 2000 to $20.7 \%$ in 2018). Areas with the highest SET rates (Delaware, the District of Columbia, and Massachusetts) also had the lowest rates of ART-conceived multiple births.

Transferring two embryos is associated with a slight increase in overall birth rate but a much greater increase in the twin birth rate compared with transferring a single embryo $(37,38)$. However, transferring two embryos sequentially (singleembryo transfer over two sequential procedures, if the first procedure did not result in a live birth) has similar cumulative live-birth rates and substantially lower twin delivery rates than transferring two embryos in a single procedure and might be a cost-effective approach; estimated costs include ART treatment and pregnancy- and infant-associated medical costs $(37,38)$. Evidence from other countries suggests that access to coverage for ART, availability of cryopreservation services, and economic and social factors regarding the number of embryos transferred per cycle can encourage SET procedures and reduce multiple births (39). In 2013, the mean health care costs to patients and insurers were estimated at $\$ 26,922$ for ARTconceived singleton deliveries, $\$ 115,238$ for ART-conceived twin deliveries, and $\$ 434,668$ for ART-conceived triplets and higher-order infants $(40)$.

The desire for twins among couples experiencing infertility and their perception that the benefits of a multiple-gestation pregnancy (compared with no pregnancy) outweigh the risks (41-43) might partially explain why twin rates remain high. Therefore, understanding the perspective of couples undergoing infertility treatments regarding multiple-gestation pregnancies and multiple births is important. Patient education focusing on maternal and perinatal morbidity and mortality and the economic costs of twin gestations has been effective in reducing the preference for twins among patients undergoing ART (44-46).

\section{Low Birthweight, Preterm Birth, and Small for Gestational Age Among ART Infants}

In the United States, although rates of ART-conceived preterm and low birthweight infants have been declining steadily, the percentage of infants born with low birthweight and born preterm remained higher among ART-conceived infants (18.3\% and $26.1 \%$, respectively) than among all infants (8.3\% and $10.0 \%$, respectively). In addition, preterm and low birthweight rates varied more widely across areas among ARTconceived infants than among all infants. 
Fertility treatments, both ART and non-ART, contribute substantially to preterm birth, which is a leading cause of infant morbidity and mortality $(33,47-49)$. The health risks associated with preterm birth have contributed to increased health care costs. In 2016, the societal economic cost associated with one infant born preterm in the United States was estimated at $\$ 64,815(33,50)$, which translates into approximately $\$ 1.3$ billion for 19,511 ARTconceived infants born preterm in 2018 .

Although ART-conceived singletons had higher rates of preterm birth $(14.9 \%)$ than singletons among the general population $(8.3 \%)$, SGA rates were lower among the former. Studies have indicated that SGA risks among ARTconceived infants might be lower than among non-ART infants and also might vary by type of ART performed (51-53). ART singletons from fresh-embryo transfers had higher risks for SGA than non-ART singletons, whereas ART singletons from frozen-embryo transfers had lower risks for SGA than nonART singletons (52). In a more recent study, SGA rates were lower among ART singletons from frozen-embryo transfers than singletons from both fresh-embryo transfers and infants conceived naturally; genetic factors as well as the freeze-thaw process might explain these differences (53). More research is needed to better understand the risks for SGA among ARTconceived infants and how these risks might vary by the type of ART cycle performed.

Use of ART only partially explains the overall prevalence of multiple births in the United States. Other factors influencing multiple births include advanced maternal age at conception and the use of non-ART fertility treatments $(33,54-56)$. The risk for multiple gestations associated with non-ART fertility treatments (i.e., controlled ovarian stimulation and ovulation induction coupled with timed intercourse or intrauterine insemination) is less well documented than that associated with ART procedures; fertility clinics are only required to report data on ART use to NASS. However, research suggests that non-ART fertility treatments contribute to a larger percentage of multiple births than ART. In 2015, approximately $17 \%$ of multiple births in the United States were attributable to IVF fertility treatments, whereas $29 \%$ were attributable to non-IVF fertility treatments $(57,58)$.

Additional efforts are needed to monitor the use of non-ART fertility treatments and their role in multiple births, particularly because the ability to control the occurrence of a multiple birth is more challenging when using non-ART fertility treatments (54). CDC is monitoring the prevalence of ART and non-ART fertility treatment use and resultant outcomes among women who had live births in states participating in the Pregnancy Risk Assessment Monitoring System (59,60).

\section{Limitations}

The findings in this report are subject to at least seven limitations. First, the procedure-specific use rates might be higher than the actual per-patient use rates. ART surveillance data were reported for each ART procedure performed rather than for each patient who used ART, and patients can undergo more than one procedure during the reporting period. In addition, the procedure-specific use rates are standardized by the size of the female population aged 15-44 years and do not account for certain ART users who are aged $>44$ years. Second, when comparisons are made between ART-conceived births and all births, all births also include ART-related births. Third, preterm birth, low birthweight, and SGA could be associated with factors contributing to underlying infertility or other maternal or paternal factors and not necessarily ART procedures. Fourth, approximately $9 \%$ of fertility clinics that performed ART in 2018 did not report their data to CDC. Although these clinics might have had results differing from reporting clinics, typically they are smaller and represent approximately $2 \%$ of all ART cycles performed in the United States (1). Fifth, gestational age is computed for ART infants conceived with frozen embryos by subtracting 17 days (to allow for an average of 3 days in embryo culture) from the date of transfer. However, many frozen-embryo transfers use blastocyst embryos (approximately 5 days of embryo culture), which might slightly underestimate gestational age. Sixth, SET rates cannot be compared with elective single embryo transfer (eSET) rates from previous reports (2016 and prior) because of differences in definition between eSET (a procedure in which one embryo is selected for transfer from a larger number of available embryos, and the remaining embryos are cryopreserved) and SET (a procedure in which one embryo is transferred regardless of the number of available embryos). In addition, in previous reports, eSET rates were reported only for procedures in which patients were using their own fresh eggs. Finally, the number of ART procedures reported for 2018 included all procedures in which banking was not performed, including procedures with frozen eggs that were thawed. Therefore, comparisons with previous years (2015 and prior) in which procedures using thawed eggs were excluded from analyses should be made with caution.

\section{Conclusion}

Since 1995, the number of ART procedures performed and the number of infants born as a result have more than tripled in the United States. With this increasing use, ART-conceived infants represented approximately $2 \%$ of infants born in 2018 
in the United States and contributed to approximately $4 \%$ and $5 \%$ of all low birthweight and preterm births, respectively. Although the percentage of all multiple births including twins, triplets, and higher-order multiples has decreased among ART-conceived infants since 2000, the percentage of twins remains high (21\%). Because multiple births have higher rates of preterm birth than singleton births, ART has a disproportionate impact on poor birth outcomes. This report provides information that allows state health departments to monitor and inform patients of the extent of ART use and ART-related adverse perinatal outcomes in their regions and take action to initiate programs and policies that improve access to ART treatments and reduce the adverse effects of ART multiple births. A state-specific website that presents key ART success rates and other statistics is available at https://www.cdc. gov/art/state-specific-surveillance/index.html.

\section{Conflict of Interest}

All authors have completed and submitted the International Committee of Medical Journal Editors form for disclosure of potential conflicts of interest. No potential conflicts of interest were disclosed.

\section{References}

1. CDC. 2018 assisted reproductive technology fertility clinic success rates report. Atlanta, GA: US Department of Health and Human Services, CDC; 2020. https://www.cdc.gov/art/reports/2018/fertility-clinic.html

2. CDC. 1995 assisted reproductive technology fertility clinic success rates report. Atlanta, GA: US Department of Health and Human Services, CDC; 1997.

3. Penzias A, Bendikson K, Butts S, et al.; Practice Committee of the American Society for Reproductive Medicine. Guidance on the limits to the number of embryos to transfer: a committee opinion. Fertil Steril 2017;107:901-3. https://doi.org/10.1016/j.fertnstert.2017.02.107

4. Di Tommaso M, Sisti G, Colombi I, et al. Influence of assisted reproductive technologies on maternal and neonatal outcomes in early preterm deliveries. J Gynecol Obstet Hum Reprod 2019;48:845-8. PMID:30898633 https://doi.org/10.1016/j.jogoh.2019.03.008

5. Le Ray C, Pelage L, Seco A, et al.; Epimoms Study Group. Risk of severe maternal morbidity associated with in vitro fertilisation: a populationbased study. BJOG 2019;126:1033-41. PMID:30801948 https://doi. org/10.1111/1471-0528.15668

6. Santana DS, Surita FG, Cecatti JG. Multiple pregnancy: epidemiology and association with maternal and perinatal morbidity. Rev Bras Ginecol Obstet 2018;40:554-62. PMID:30231294 https://doi. org/10.1055/s-0038-1668117

7. MacKay AP, Berg CJ, King JC, Duran C, Chang J. Pregnancy-related mortality among women with multifetal pregnancies. Obstet Gynecol 2006;107:563-8. PMID:16507925 https://doi.org/10.1097/01. AOG.0000200045.91015.c6

8. Fountain C, Zhang Y, Kissin DM, et al. Association between assisted reproductive technology conception and autism in California, 1997-2007. Am J Public Health 2015;105:963-71. PMID:25790396 https://doi. org/10.2105/AJPH.2014.302383

9. Boulet SL, Kirby RS, Reefhuis J, et al.; States Monitoring Assisted Reproductive Technology (SMART) Collaborative. Assisted reproductive technology and birth defects among liveborn infants in Florida, Massachusetts, and Michigan, 2000-2010. JAMA Pediatr 2016;170:e154934. PMID:27043648 https://doi.org/10.1001/ jamapediatrics.2015.4934
10. Boulet SL, Schieve LA, Nannini A, et al. Perinatal outcomes of twin births conceived using assisted reproduction technology: a populationbased study. Hum Reprod 2008;23:1941-8. PMID:18487216 https:// doi.org/10.1093/humrep/den169

11. Dawson AL, Tinker SC, Jamieson DJ, et al.; National Birth Defects Prevention Study. Twinning and major birth defects, National Birth Defects Prevention Study, 1997-2007. J Epidemiol Community Health 2016;70:1114-21. PMID:27325867 https://doi.org/10.1136/ jech-2015-206302

12. Qin JB, Sheng XQ, Wu D, et al. Worldwide prevalence of adverse pregnancy outcomes among singleton pregnancies after in vitro fertilization/intracytoplasmic sperm injection: a systematic review and meta-analysis. Arch Gynecol Obstet 2017;295:285-301. PMID:27896474 https://doi.org/10.1007/s00404-016-4250-3

13. Martin AS, Chang J, Zhang Y, et al.; States Monitoring Assisted Reproductive Technology (SMART) Collaborative. Perinatal outcomes among singletons after assisted reproductive technology with singleembryo or double-embryo transfer versus no assisted reproductive technology. Fertil Steril 2017;107:954-60. PMID:28292615 https:// doi.org/10.1016/j.fertnstert.2017.01.024

14. Farr SL, Schieve LA, Jamieson DJ. Pregnancy loss among pregnancies conceived through assisted reproductive technology, United States, 1999-2002. Am J Epidemiol 2007;165:1380-8. PMID:17351291 https://doi.org/10.1093/aje/kwm035

15. Hipp H, Crawford S, Kawwass JF, Chang J, Kissin DM, Jamieson DJ. First trimester pregnancy loss after fresh and frozen in vitro fertilization cycles. Fertil Steril 2016;105:722-8. PMID:26627121 https://doi. org/10.1016/j.fertnstert.2015.11.012

16. National Center for Health Statistics. Vital statistics data available. Natality public use file and CD-ROM. Hyattsville, MD: National Center for Health Statistics.

17. Martin JA, Hamilton BE, Osterman MJK, Driscoll AK. Births: Final Data for 2018. Natl Vital Stat Rep 2019;68:1-47. PMID:32501202

18. US Census Bureau. Annual estimates of the resident population for selected age groups by sex for the United States, states, counties, and Puerto Rico Commonwealth and municipios: April 1, 2010 to July 1, 2018. Washington, DC: US Census Bureau, Population Division; 2018. https://data.census.gov/cedsci/table?q=United\%20States\&g=0100000 US\&tid=ACSST1Y2018.S0101\&vintage $=2018$

19. Martin JA, Osterman MJ, Kirmeyer SE, Gregory EC. Measuring gestational age in vital statistics data: transitioning to the obstetric estimate. Natl Vital Stat Rep 2015;64:1-20. PMID:26047089

20. Talge NM, Mudd LM, Sikorskii A, Basso O. United States birth weight reference corrected for implausible gestational age estimates. Pediatrics 2014;133:844-53. PMID:24777216 https://doi.org/10.1542/ peds.2013-3285

21. Sunderam S, Kissin DM, Zhang Y, et al. Assisted reproductive technology surveillance-United States, 2017. MMWR Surveill Summ 2020;69:1-20. PMID:33332294 https://doi.org/10.15585/mmwr.ss6909a1

22. Henne MB, Bundorf MK. Insurance mandates and trends in infertility treatments. Fertil Steril 2008;89:66-73. PMID:17482603 https://doi. org/10.1016/j.fertnstert.2007.01.167

23. Hamilton BH, McManus B. The effects of insurance mandates on choices and outcomes in infertility treatment markets. Health Econ 2012;21:994-1016. PMID:21905150 https://doi.org/10.1002/ hec. 1776

24. Boulet SL, Crawford S, Zhang Y, et al.; States Monitoring ART Collaborative. Embryo transfer practices and perinatal outcomes by insurance mandate status. Fertil Steril 2015;104:403-9.e1. PMID:26051096 https://doi.org/10.1016/j.fertnstert.2015.05.015

25. Zagadailov P, Seifer DB, Shan H, Zarek SM, Hsu AL. Do state insurance mandates alter ICSI utilization? Reprod Biol Endocrinol 2020;18:33. PMID:32334609 https://doi.org/10.1186/s12958-020-00589-w 
26. RESOLVE. Infertility coverage in your state. McLean, VA: RESOLVE. https://resolve.org/what-are-my-options/insurance-coverage/ infertility-coverage-state/

27. Dieke AC, Zhang Y, Kissin DM, Barfield WD, Boulet SL. Disparities in assisted reproductive technology utilization by race and ethnicity, United States, 2014: a commentary. J Womens Health (Larchmt) 2017;26:605-8. PMID:28586255 https://doi.org/10.1089/jwh.2017.6467

28. Quinn M, Fujimoto V. Racial and ethnic disparities in assisted reproductive technology access and outcomes. Fertil Steril 2016;105:1119-23. PMID:27054308 https://doi.org/10.1016/j. fertnstert.2016.03.007

29. Practice Committee of Society for Assisted Reproductive Technology; Practice Committee of American Society for Reproductive Medicine. Guidelines on number of embryos transferred. Fertil Steril 2008;90(Suppl):S163-4. PMID:19007617 https://doi.org/10.1016/j. fertnstert.2008.08.053

30. Practice Committee of the American Society for Reproductive Medicine; Practice Committee of the Society for Assisted Reproductive Technology. Guidelines on number of embryos transferred. Fertil Steril 2009;92:1518-9. PMID:19836732 https://doi.org/10.1016/j. fertnstert.2009.08.059

31. Practice Committee of the Society for Assisted Reproductive Technology; Practice Committee of the American Society for Reproductive Medicine. Guidelines on number of embryos transferred. Fertil Steril 2006;86(Suppl 1):S51-2. PMID:17055845 https://doi.org/10.1016/j. fertnstert.2006.07.1473

32. Practice Committee of the American Society for Reproductive Medicine; Practice Committee of the Society for Assisted Reproductive Technology. Criteria for number of embryos to transfer: a committee opinion. Fertil Steril 2013;99:44-6. PMID:23095140 https://doi.org/10.1016/j. fertnstert.2012.09.038

33. Institute of Medicine, Board on Health Sciences Policy, Committee on Understanding Premature Birth and Assuring Healthy Outcomes. Premature birth: causes, consequences, and prevention. Behrman RE, Butler AS, eds. Washington, DC: National Academies Press; 2007.

34. Practice Committee of American Society for Reproductive Medicine. Multiple gestation associated with infertility therapy: an American Society for Reproductive Medicine Practice Committee opinion. Fertil Steril 2012;97:825-34. PMID:22192352 https://doi.org/10.1016/j. fertnstert.2011.11.048

35. Kissin DM, Kulkarni AD, Kushnir VA, Jamieson DJ; National ART Surveillance System Group. Number of embryos transferred after in vitro fertilization and good perinatal outcome. Obstet Gynecol 2014;123:239-47. PMID:24402601 https://doi.org/10.1097/ AOG.0000000000000106

36. Wright VC, Schieve LA, Reynolds MA, Jeng G. Assisted reproductive technology surveillance-United States, 2000. MMWR Surveill Summ 2003;52:1-16. PMID:14532867

37. Luke B, Brown MB, Wantman E, et al. Application of a validated prediction model for in vitro fertilization: comparison of live birth rates and multiple birth rates with 1 embryo transferred over 2 cycles vs 2 embryos in 1 cycle. Am J Obstet Gynecol 2015;212:676.e1-7. PMID:25683965 https://doi.org/10.1016/j.ajog.2015.02.005

38. Crawford S, Boulet SL, Mneimneh AS, et al. Costs of achieving live birth from assisted reproductive technology: a comparison of sequential single and double embryo transfer approaches. Fertil Steril 2016;105:444-50. PMID:26604068 https://doi.org/10.1016/j.fertnstert.2015.10.032

39. Maheshwari A, Griffiths S, Bhattacharya S. Global variations in the uptake of single embryo transfer. Hum Reprod Update 2011;17:107-20. PMID:20634207 https://doi.org/10.1093/humupd/dmq028

40. Lemos EV, Zhang D, Van Voorhis BJ, Hu XH. Healthcare expenses associated with multiple vs singleton pregnancies in the United States. Am J Obstet Gynecol 2013;209:586.e1-11. PMID:24238479 https:// doi.org/10.1016/j.ajog.2013.10.005
41. Grobman WA, Milad MP, Stout J, Klock SC. Patient perceptions of multiple gestations: an assessment of knowledge and risk aversion. Am J Obstet Gynecol 2001;185:920-4. PMID:11641679 https://doi. org/10.1067/mob.2001.117305

42. Blennborn M, Nilsson S, Hillervik C, Hellberg D. The couple's decisionmaking in IVF: one or two embryos at transfer? Hum Reprod 2005;20:1292-7. PMID:15734759 https://doi.org/10.1093/humrep/deh785

43. Pinborg A, Loft A, Schmidt L, Andersen AN. Attitudes of IVF/ICSI-twin mothers towards twins and single embryo transfer. Hum Reprod 2003;18:621-7. PMID:12615836 https://doi.org/10.1093/humrep/deg145

44. Ryan GL, Sparks AE, Sipe CS, Syrop CH, Dokras A, Van Voorhis BJ. A mandatory single blastocyst transfer policy with educational campaign in a United States IVF program reduces multiple gestation rates without sacrificing pregnancy rates. Fertil Steril 2007;88:354-60. PMID:17490657 https://doi.org/10.1016/j.fertnstert.2007.03.001

45. Hope N, Rombauts L. Can an educational DVD improve the acceptability of elective single embryo transfer? A randomized controlled study. Fertil Steril 2010;94:489-95. PMID:19442970 https://doi.org/10.1016/j. fertnstert.2009.03.080

46. Sunderam S, Boulet SL, Jamieson DJ, Kissin DM. Effects of patient education on desire for twins and use of elective single embryo transfer procedures during ART treatment: A systematic review. Reprod Biomed Soc Online 2018;6:102-19. PMID:30761357 https://doi.org/10.1016/j. rbms.2018.10.017

47. Callaghan WM, MacDorman MF, Rasmussen SA, Qin C, Lackritz EM. The contribution of preterm birth to infant mortality rates in the United States. Pediatrics 2006;118:1566-73. PMID:17015548 https://doi. org/10.1542/peds.2006-0860

48. Tanner K, Sabrine N, Wren C. Cardiovascular malformations among preterm infants. Pediatrics 2005;116:e833-8. PMID:16322141 https:// doi.org/10.1542/peds.2005-0397

49. Rasmussen SA, Moore CA, Paulozzi LJ, Rhodenhiser EP. Risk for birth defects among premature infants: a population-based study. J Pediatr 2001;138:668-73. PMID:11343041 https://doi.org/10.1067/ mpd.2001.112249

50. Waitzman N, Jalali A. Updating national preterm birth costs to 2016 with separate estimates for individual states. Final report to the March of Dimes. https://www.marchofdimes.org/peristats/documents/Cost_ of_Prematurity_2019.pdf

51. D'Angelo DV, Whitehead N, Helms K, Barfield W, Ahluwalia IB. Birth outcomes of intended pregnancies among women who used assisted reproductive technology, ovulation stimulation, or no treatment. Fertil Steril 2011;96:314-320.e2. PMID:21718990 https://doi.org/10.1016/j. fertnstert.2011.05.073

52. Dunietz GL, Holzman C, Zhang Y, et al. Assisted reproductive technology and newborn size in singletons resulting from fresh and cryopreserved embryos transfer. PLoS One 2017;12:e0169869. Erratum in: PLoS One 2018;13:e0196767. PMID:28114395 https://doi. org/10.1371/journal.pone.0169869

53. Terho AM, Pelkonen S, Opdahl S, et al. High birth weight and largefor-gestational-age in singletons born after frozen compared to fresh embryo transfer, by gestational week: a Nordic register study from the CoNARTaS group. Hum Reprod 2021;36:1083-92. PMID:33416878 https://doi.org/10.1093/humrep/deaa304

54. Kulkarni AD, Jamieson DJ, Jones HW Jr, et al. Fertility treatments and multiple births in the United States. N Engl J Med 2013;369:2218-25. PMID:24304051 https://doi.org/10.1056/NEJMoa1301467

55. Martin JA, Hamilton BE, Osterman MJ. Three decades of twin births in the United States, 1980-2009. NCHS Data Brief 2012;80:1-8. PMID:22617378

56. Adashi EY, Gutman R. Delayed childbearing as a growing, previously unrecognized contributor to the national plural birth excess. Obstet Gynecol 2018;132:999-1006. PMID:30204699 https://doi. org/10.1097/AOG.0000000000002853 
57. Sunderam S, Kissin DM, Crawford SB, Kulkarni AD, Boulet SL. Overview of 2015 U.S. assisted reproductive technology (ART) treatment outcomes and contribution of ART and non-ART fertility treatments to U.S. multiple births. Fertil Steril 2017;108:e97-8. https:// doi.org/10.1016/j.fertnstert.2017.07.297

58. Kulkarni AD, Adashi EY, Jamieson DJ, Crawford SB, Sunderam S, Kissin DM. Affordability of fertility treatments and multiple births in the United States. Paediatr Perinat Epidemiol 2017;31:438-48. PMID:28762537 https://doi.org/10.1111/ppe.12383
59. Barradas DT, Barfield WD, Wright V, D'Angelo D, Manning SE, Schieve LA. Assessment of assisted reproductive technology use questions: Pregnancy Risk Assessment Monitoring System Survey, 2004. Public Health Rep 2012;127:516-23. PMID:22942469 https://doi. org/10.1177/003335491212700507

60. Sanders J, Simonsen S, Porucznik CA, Baksh L, Stanford JB. Use of fertility treatments in relation to the duration of pregnancy attempt among women who were trying to become pregnant and experienced a live birth. Matern Child Health J 2014;18:258-67. PMID:23584927 https://doi.org/10.1007/s10995-013-1262-5 


The Morbidity and Mortality Weekly Report (MMWR) Series is prepared by the Centers for Disease Control and Prevention (CDC) and is available free of charge in electronic format. To receive an electronic copy each week, visit MMWR at https://www.cdc.gov/mmwr/index.html.

Readers who have difficulty accessing this PDF file may access the HTML file at https://www.cdc.gov/mmwr/volumes/71/ss/ss7104a1.htm?s_ cid=ss7104a1_w. Address all inquiries about the $M M W R$ Series to Editor-in-Chief, MMWR Series, Mailstop V25-5, CDC, 1600 Clifton Rd., N.E., Atlanta, GA 30329-4027 or to mmwrq@cdc.gov.

All material in the MMWR Series is in the public domain and may be used and reprinted without permission; citation as to source, however, is appreciated. MMWR and Morbidity and Mortality Weekly Report are service marks of the U.S. Department of Health and Human Services.

Use of trade names and commercial sources is for identification only and does not imply endorsement by the U.S. Department of Health and Human Services.

References to non-CDC sites on the Internet are provided as a service to $M M W R$ readers and do not constitute or imply endorsement of these organizations or their programs by CDC or the U.S. Department of Health and Human Services. CDC is not responsible for the content of these sites. URL addresses listed in $M M W R$ were current as of the date of publication.

ISSN: 1546-0738 (Print) 